\title{
Tentative model of crustal structure of the Central Indian Ocean deformation as inferred from spectral and Werner deconvolution techniques of magnetic data
}

\author{
R. Divya Bharathi ${ }^{1}$, M. Subrahmanyam ${ }^{1, *}$, D. S. Ramesh ${ }^{2}$ and S. Rajendran ${ }^{3}$ \\ ${ }^{1}$ Department of Geophysics, Andhra University, Visakhapatnam 530 003, India \\ ${ }^{2}$ Indian Institute of Geomagnetism, Kalamboli Highway, New Panvel, New Mumbai 410 218, India \\ ${ }^{3}$ Department of Earth Sciences, Kerala University of Fisheries and Ocean Studies, Panangad, Cochin 682 506, India
}

\begin{abstract}
Nine total magnetic profiles in the $S-N$ direction of the Central Indian Ocean (CIO) have been interpreted with spectral and Werner deconvolution techniques to obtain approximate depths to shallow (magnetic basement) and deeper (Curie isotherm) interfaces of the oceanic crust. From these preliminary depths, tentative crustal models for all these nine profiles are prepared. These tentative models of the area show varying thickness of the oceanic crust, supporting the deformation history. It is inferred from these tentative models that deformation extends beyond the zone identified south of $8^{\circ} \mathrm{S}$ latitude. There is good agreement between the spectral and Werner deconvolution depths.
\end{abstract}

Keywords: Deformation zone, deconvolution techniques, magnetic anomaly, oceanic crust, tentative model.

THE status of the Central Indian Ocean (CIO) as a zone of deformation was first noted from intraplate seismicity ${ }^{1}$. The zone of intraplate deformation in the CIO is one of the best documented tectonic elements (Figure 1). Thrust and strike-slip motion at upper mantle depths on E-W and $\mathrm{N}-\mathrm{S}$ fault planes respectively, testify to brittle failure in the lithosphere ${ }^{2}$. Maximum horizontal compressive stress vectors imply sinistral transpression across the fracture zones (FZs) throughout the area ${ }^{3,4}$. The continent-continent collision to the north of the Indian shield ${ }^{5}$, continuous generation of new oceanic lithosphere along Carlsberg ridge-Central Indian Ridge-Southeast Indian Ridge and subsequent events unifying the Indian and Australian Plates are the causes of instability of the CIO lithosphere ${ }^{6}$. The intraplate deformation is revealed well in seismic reflection profiles and is characterized by a diffuse zone of large magnitude seismicity ${ }^{3,7}$, localized high heat flow ${ }^{8}$ and gravity as well as geoid anomalies ${ }^{9-12}$.

*For correspondence. (e-mail: smangalampalli@rediffmail.com)
The intraplate deformation in the northern Indian Ocean is now well documented from geophysical data $^{2,3,10,12-14}$. It has been explained in terms of diffuse plate boundary between the Indian and Australian plates $^{15-18}$. The deformation, although dominantly compressive, also has a strike-slip component ${ }^{11,19}$.

The tectonic deformation is found to be occurring on two spatial scales. First, there are long-wavelength (100$300 \mathrm{~km}$ ) undulations of oceanic magnetic basement and overlying sediments. Second, there are shorter wavelength $(5-10 \mathrm{~km})$ reverse faults and associated folds due to buckling. The former penetrate all through the deformed oceanic crust ${ }^{12}$. The relationship between the thickness of the brittle layer and wavelength of the fold shows that the wavelength is seven times the thickness of the brittle layer ${ }^{19}$. Detailed analysis of the seismic reflection profiles $(\approx 5370 \mathrm{~km})$ of the Central Indian Ocean Basin (CIOB) deformation zone confirmed the existence of long-wavelength (150-300 km) antiformal basement structures with $1-2 \mathrm{~km}$ relief, tight folding and highangle faulting (5-20 km long) of the oceanic basement and overlying sediments ${ }^{20}$.

The average heat flow value in the CIOB is $30 \mathrm{~mW} / \mathrm{m}^{2}$, which is higher than what is expected from the cooling models ${ }^{9,12,13,21}$. The CIOB is the largest of all the Indian Ocean basins that has been studied in detail for its mineral potential. Extensive exploration has led to the delineation of manganese nodule resources of about 1300 million metric tonnes ${ }^{22}$. The field demarcated as the Indian Ocean Nodule Field (IONF), occurs between $10^{\circ}-16^{\circ} 30^{\prime} \mathrm{S}$ and $72^{\circ}-80^{\circ} \mathrm{E}$, where average water depth is $5000 \mathrm{~m}$. This area holds the mineable ferromanganese deposits in the Indian Ocean ${ }^{23-25}$.

We have carried out magnetic anomaly study in a part of the deformation zone of the CIO lying between $6^{\circ} \mathrm{N}-$ $18^{\circ} \mathrm{S}$ and $76^{\circ}-84^{\circ} \mathrm{E}$. As a preliminary analysis, data have been interpreted by spectral and Werner deconvolution methods to arrive at a tentative crustal depth model. The performance of these two techniques has been discussed in detail by Subrahmanyam and Gebissa ${ }^{26}$. 


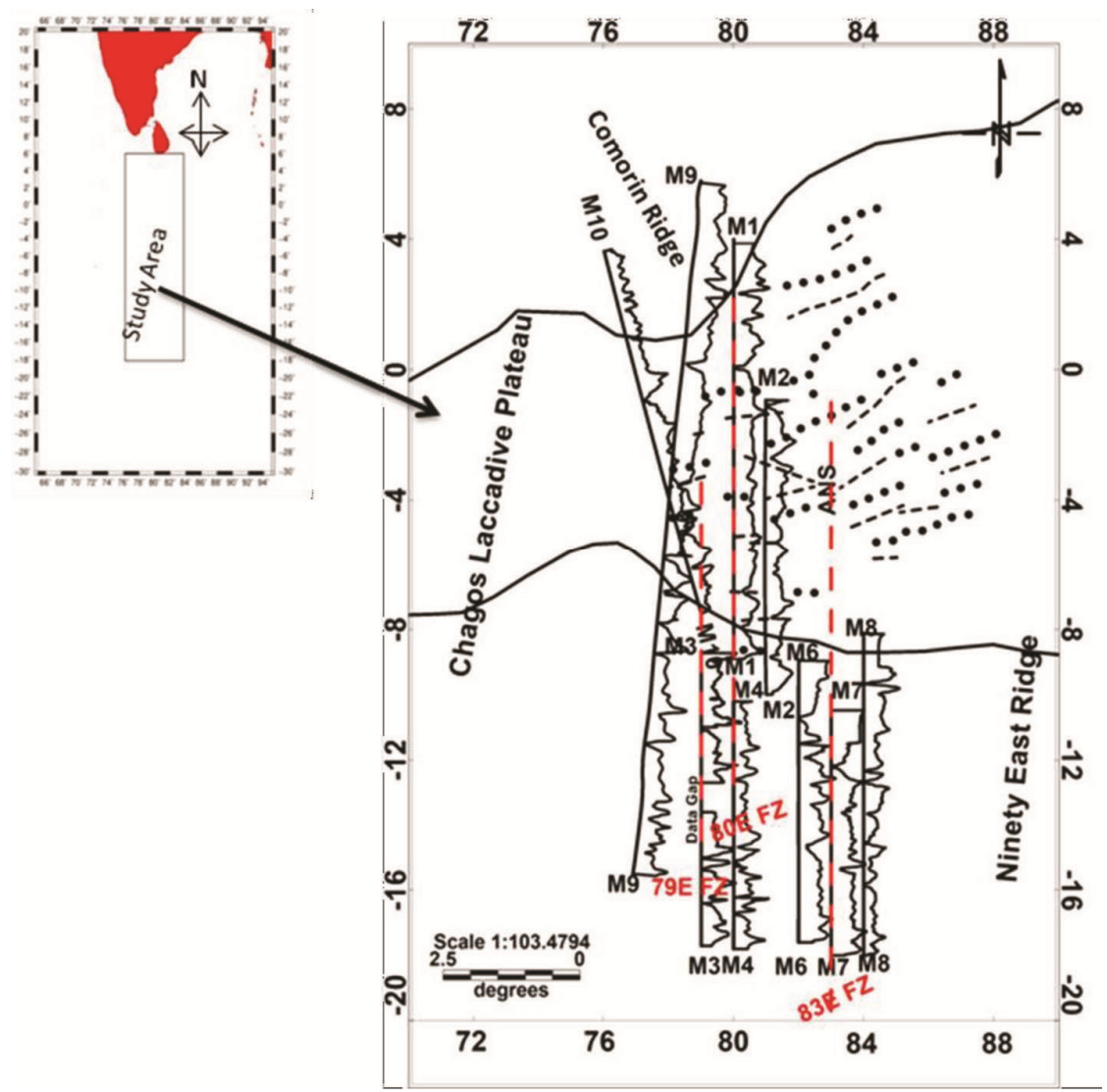

Figure 1. The intraplate deformation zone area between thick solid curves that divides the Indian and Australian plates ${ }^{6}$. Black dotted and dashed lines are the crest and troughs respectively ${ }^{2}$. ANS is the Afanasy Nikitin Seamount. M1M1 to M4M4 and M6M6 to M10M10 are the locations of the magnetic profiles with magnetic anomalies. The red dashed lines are fracture zones (FZ) in the study area ${ }^{20}$.

\section{Data}

Figure 1 shows the locations of the magnetic data profiles of this study. Ship-borne magnetic data along nine magnetic profiles were collected during 1982-84 and digitized at 1 nautical mile interval by Rajendran ${ }^{27}$. The amplitude of the total field magnetic anomaly varied in the range -900 to $500 \mathrm{nT}$. The shape of the anomalies depends on the thickness and susceptibility. The data were interpreted for the age of the oceanic crust ${ }^{27}$.

The magnetic anomalies were characterized by medium amplitude and short wavelengths in conformity with the spreading anomalies normally observed in abyssal plains of the world oceans. The large-magnitude magnetic anomalies $(\sim 800 \mathrm{nT})$ observed at the northern end of profiles M3M3, M6M6 and M7M7 may be due to $79^{\circ} \mathrm{E}$ (adjacent to $\mathrm{M} 3 \mathrm{M} 3$ ) and $83^{\circ} \mathrm{E}$ (between M6M6 and M7M7) FZs.

\section{Methodology and processing}

The magnetic anomalies were interpreted with spectral and Werner deconvolution methods. Both these methods give the depths to the interfaces ${ }^{26}$.
Each magnetic profile was divided into a number of segments. The segmentation of anomalies was done with the knowledge of the magnetic lineations (age anomalies). Each segment overlapped the adjacent segment by $10-15 \%$.

The magnetic anomalies (space domain) were transformed into frequency domain using fast Fourier transform. Amplitude versus frequency of the transformed data was plotted. The graph showed a straight line pattern and slope of the straight line gave the depth to the top of the layer (interface) or source. Using this, depth to the interface was determined. For determining the deeper marker (Curie isotherms), the anomalies were filtered with low-pass filter to extract the long-wavelength anomalies which may be due to deeper interfaces. To extract the long-wavelength anomalies, several frequencies $(0.04,0.06,0.08,0.1$ and $0.12 \mathrm{~Hz})$ were applied. The low-pass filter of $0.1 \mathrm{~Hz}$ was found to yield the appropriate representative deeper interface of the oceanic crust. To determine shallow and deeper interfaces with Werner deconvolution method, unfiltered and filtered magnetic anomalies respectively, of each segment were used. The same procedure was carried out for all the profiles and tentative models were developed. 
Table 1. Depths determined from spectral and Werner deconvolution techniques for each segment of profiles M1M1, M2M2, M3M3, M4M4, M6M6, M7M7, M8M8, M9M9 and M10M10

\begin{tabular}{|c|c|c|c|c|c|c|c|c|c|c|}
\hline \multirow{3}{*}{$\begin{array}{l}\text { Profile } \\
\text { no. }\end{array}$} & \multirow{3}{*}{$\begin{array}{l}\text { Segment } \\
\text { no. }\end{array}$} & \multirow{3}{*}{$\begin{array}{l}\text { Distance } \\
(\mathrm{km})\end{array}$} & \multirow{3}{*}{$\begin{array}{l}\text { Bathy- } \\
\text { metry }(\mathrm{km}) \\
\text { minimum- } \\
\text { maximum }\end{array}$} & \multirow{3}{*}{$\begin{array}{l}\text { Average depth } \\
\text { to the bottom } \\
\text { of the sediment } \\
\text { layer (bathy }+ \\
\text { sediment } \\
\text { thickness; km) }\end{array}$} & \multirow{2}{*}{\multicolumn{2}{|c|}{ Spectral depth $(\mathrm{km})$}} & \multirow{2}{*}{\multicolumn{2}{|c|}{ Werner depths (km) }} & \multicolumn{2}{|c|}{ Remarks } \\
\hline & & & & & & & & & Results of & \\
\hline & & & & & Unfiltered & Filtered & Unfiltered & Filtered & study & Scrutton $^{2}$ \\
\hline \multirow[t]{11}{*}{$\begin{array}{l}\mathrm{M} 1 \mathrm{M} 1 \\
\left(9^{\circ} \mathrm{S}-4^{\circ} \mathrm{N}\right. \\
\text { along } \\
\left.\sim 80^{\circ} \mathrm{E}\right)\end{array}$} & $\begin{array}{l}\text { M1M1-1 } \\
\left(9^{\circ}-7.6^{\circ} \mathrm{S}\right)\end{array}$ & $0-150$ & $4.69-5.5$ & 5.10 & 6.09 & 11.25 & $\begin{array}{c}6.4(20), \\
5.4(80) \\
6(155)\end{array}$ & $\begin{array}{c}10(0), \\
10.4(80), \\
11.4(120)\end{array}$ & $\begin{array}{l}\text { Crest } \\
\left(8.35^{\circ} \mathrm{S}\right)\end{array}$ & $\begin{array}{l}\text { Crest } \\
\left(8.6^{\circ} \mathrm{S}\right), \\
\text { Trough } \\
\left(7.7^{\circ} \mathrm{S}\right)\end{array}$ \\
\hline & $\begin{array}{l}\text { M1M1-2 } \\
\left(7.8^{\circ}-6.1^{\circ} \mathrm{S}\right)\end{array}$ & $138-323$ & $4.9-5.5$ & 5.20 & 6.33 & 10.6 & $\begin{array}{l}6.4(230), \\
6.5(260), \\
6.4(300)\end{array}$ & $\begin{array}{c}11(150), \\
11.5(240), \\
9.6(290)\end{array}$ & $\begin{array}{l}\text { Trough } \\
\left(6.8^{\circ} \mathrm{S}\right)\end{array}$ & $\begin{array}{l}\text { Trough } \\
\left(6.8^{\circ} \mathrm{S}\right)\end{array}$ \\
\hline & $\begin{array}{l}\text { M1M1-3 } \\
\left(6.2^{\circ}-4.7^{\circ} \mathrm{S}\right)\end{array}$ & $310-480$ & $5.16-5.20$ & 5.18 & 5.83 & 11.3 & $\begin{array}{l}6.6(340), \\
6.2(400)\end{array}$ & $10(380)$ & & \\
\hline & $\begin{array}{l}\text { M1M1-4 } \\
\left(4.6^{\circ}-3.8^{\circ} \mathrm{S}\right)\end{array}$ & $473-578$ & $4.55-5.21$ & 4.88 & 6.01 & 11.06 & $\begin{array}{l}6.5(520) \\
5.6(560)\end{array}$ & $\begin{array}{l}11.5(513) \\
11.8(548)\end{array}$ & & $\begin{array}{l}\text { Trough } \\
\left(4.2^{\circ} \mathrm{S}\right), \\
\text { Crest }\left(3.8^{\circ} \mathrm{S}\right)\end{array}$ \\
\hline & $\begin{array}{l}\text { M1M1-5 } \\
\left(3.9^{\circ}-1.9^{\circ} \mathrm{S}\right)\end{array}$ & $568-780$ & $4.79-5.06$ & 4.93 & 6.40 & 11.8 & $\begin{array}{l}6.6(590), \\
6.6(670), \\
6.6(740)\end{array}$ & $\begin{array}{c}10.8(593) \\
12(738)\end{array}$ & & \\
\hline & $\begin{array}{l}\text { M1M1-6 } \\
\left(2.2^{\circ}-0.4^{\circ} \mathrm{N}\right)\end{array}$ & $760-1048$ & $4.61-4.91$ & 4.71 & 6.42 & 11.9 & $\begin{array}{l}6.8(770), \\
5.6(860), \\
6.8(950), \\
5.2(980)\end{array}$ & $\begin{array}{c}11(810), \\
11.8(858) \\
11.4(920) \\
10.5(980)\end{array}$ & $\begin{array}{l}\text { Trough } \\
\left(1.44^{\circ} \mathrm{S}\right)\end{array}$ & $\begin{array}{l}\text { Trough } \\
\left(1.5^{\circ} \mathrm{S}\right), \\
\text { Crest } \\
\left(0.6^{\circ} \mathrm{S}\right)\end{array}$ \\
\hline & $\begin{array}{l}\text { M1M1-7 } \\
\left(0.3^{\circ}-6^{\circ} \mathrm{N}\right)\end{array}$ & $\begin{array}{c}1037- \\
1180\end{array}$ & $3.53-4.69$ & 4.13 & 5.80 & 9.62 & $\begin{array}{l}5.2(1110), \\
5.6(1180)\end{array}$ & $\begin{array}{c}10.5(1067), \\
10.5(1107), \\
10(1127)\end{array}$ & & \\
\hline & $\begin{array}{l}\text { M1M1-8 } \\
\left(1.5^{\circ}-0.2^{\circ} \mathrm{N}\right)\end{array}$ & $\begin{array}{l}1161- \\
1247\end{array}$ & $3.54-4.6$ & 4.12 & 6.00 & 12.15 & $6.4(1230)$ & $10.0(1157)$ & & \\
\hline & $\begin{array}{l}\text { M1M1-9 } \\
\left(2.0^{\circ}-0.7^{\circ} \mathrm{N}\right)\end{array}$ & $\begin{array}{c}1224- \\
1294\end{array}$ & $3.48-4.4$ & 3.94 & 5.80 & 12.1 & $6.3(1310)$ & $\begin{array}{c}14.4(1191), \\
14(123), \\
13.6(1244), \\
14.4(1280)\end{array}$ & & \\
\hline & $\begin{array}{l}\text { M1M1-10 } \\
\left(2.5^{\circ}-0.9^{\circ} \mathrm{N}\right)\end{array}$ & $\begin{array}{c}1280- \\
1322\end{array}$ & $4.28-4.33$ & 5.85 & 5.60 & 9.08 & $6.4(1350)$ & 12(1305) & & \\
\hline & $\begin{array}{l}\text { M1M1-11 } \\
\left(2.9^{\circ}-4^{\circ} \mathrm{N}\right)\end{array}$ & $\begin{array}{c}1318- \\
1438\end{array}$ & $4.28-4.36$ & 6.09 & 6.50 & 9.75 & $6.4(1410)$ & $\begin{array}{c}10.8(1378), \\
9.6(1408), \\
10.2(1438)\end{array}$ & & \\
\hline \multirow[t]{6}{*}{$\begin{array}{l}\text { M2M2 } \\
\left(10^{\circ}-0^{\circ}\right. \\
\text { along } \\
\left.\sim 81^{\circ} \mathrm{E}\right)\end{array}$} & $\begin{array}{l}\text { M2M2-1 } \\
\left(10^{\circ}-8.3^{\circ} \mathrm{S}\right)\end{array}$ & $0-184$ & $5.02-5.23$ & 5.26 & 5.35 & 10.47 & $\begin{array}{l}5.4(40), \\
5.2(105), \\
5.3(115), \\
5.2(140), \\
5.6(170)\end{array}$ & $\begin{array}{c}9.4(0), \\
9(40, \\
12(105), \\
10.6(140)\end{array}$ & $\begin{array}{l}\text { Crest } \\
\left(8.7^{\circ} \mathrm{S}\right)\end{array}$ & $\begin{array}{l}\text { Crest } \\
\left(8.6^{\circ} \mathrm{S}\right)\end{array}$ \\
\hline & $\begin{array}{l}\text { M2M2-2 } \\
\left(8.4^{\circ}-6.8^{\circ} \mathrm{S}\right)\end{array}$ & $179-356$ & $5.03-5.28$ & 5.3 & 5.4 & 11.5 & $\begin{array}{l}5.8(180), \\
5.4(240), \\
5.9(290)\end{array}$ & $\begin{array}{c}10.2(200), \\
9.8(230), \\
10.2(260), \\
11.2(300)\end{array}$ & & $\begin{array}{l}\text { Trough } \\
\left(7.7^{\circ} \mathrm{S}\right)\end{array}$ \\
\hline & $\begin{array}{l}\text { M2M2-3 } \\
\left(6.84^{\circ}-\right. \\
\left.0.6^{\circ} \mathrm{S}\right)\end{array}$ & $350-484$ & $5.04-5.17$ & 5.25 & 5.76 & 10.25 & $6.2(440)$ & 10.4(450), & & \\
\hline & $\begin{array}{l}\text { M2M2-4 } \\
\left(5.72^{\circ}-\right. \\
\left.0.4^{\circ} \mathrm{S}\right)\end{array}$ & $476-620$ & $4.79-5.13$ & 5.4 & 5.85 & 12.89 & $\begin{array}{c}5.8(560) \\
6(620)\end{array}$ & $\begin{array}{l}11.6(536), \\
12.2(596)\end{array}$ & & $\begin{array}{l}\text { Trough } \\
\left(5.4^{\circ} \mathrm{S}\right), \\
\text { Crest } \\
\left(4.6^{\circ} \mathrm{S}\right)\end{array}$ \\
\hline & $\begin{array}{l}\mathrm{M} 2 \mathrm{M} 2-5 \\
\left(4.5^{\circ}-\right. \\
\left.3.31^{\circ} \mathrm{S}\right)\end{array}$ & $608-745$ & $4.76-4.97$ & 5.8 & 5.9 & 11.78 & $\begin{array}{c}6.1(670) \\
6.5(720)\end{array}$ & $\begin{array}{c}11(680), \\
11.5(710) \\
12.5(723)\end{array}$ & & $\begin{array}{l}\text { Trough } \\
(4.00 \mathrm{~S})\end{array}$ \\
\hline & $\begin{array}{l}\text { M2M2-6 } \\
\left(3.4^{\circ}-2.3^{\circ} \mathrm{S}\right)\end{array}$ & $738-859$ & $4.72-4.86$ & 4.9 & 6.3 & 12.66 & $\begin{array}{c}6.4(790) \\
6.3(820)\end{array}$ & $\begin{array}{l}11.8(740), \\
12.8(798), \\
11.5(823), \\
11.6(848)\end{array}$ & & $\begin{array}{l}\text { Trough } \\
\left(2.9^{\circ} \mathrm{S}\right)\end{array}$ \\
\hline
\end{tabular}


RESEARCH ARTICLES

Table 1. (Contd)

\begin{tabular}{|c|c|c|c|c|c|c|c|c|c|c|}
\hline \multirow{3}{*}{$\begin{array}{l}\text { Profile } \\
\text { no. }\end{array}$} & \multirow{3}{*}{$\begin{array}{l}\text { Segment } \\
\text { no. }\end{array}$} & \multirow{3}{*}{$\begin{array}{l}\text { Distance } \\
(\mathrm{km})\end{array}$} & \multirow{3}{*}{$\begin{array}{l}\text { Bathy- } \\
\text { metry (km) } \\
\text { minimum- } \\
\text { maximum }\end{array}$} & \multirow{3}{*}{$\begin{array}{l}\text { Average depth } \\
\text { to the bottom } \\
\text { of the sediment } \\
\text { layer (bathy + } \\
\text { sediment } \\
\text { thickness; km) }\end{array}$} & \multirow{2}{*}{\multicolumn{2}{|c|}{ Spectral depth $(\mathrm{km})$}} & \multirow{2}{*}{\multicolumn{2}{|c|}{ Werner depths $(\mathrm{km})$}} & \multicolumn{2}{|c|}{ Remarks } \\
\hline & & & & & & & & & \multirow{2}{*}{$\begin{array}{c}\text { Results of } \\
\text { the present } \\
\text { study }\end{array}$} & \multirow{2}{*}{$\begin{array}{l}\text { Bull and } \\
\text { Scrutton }^{2}\end{array}$} \\
\hline & & & & & Unfiltered & Filtered & Unfiltered & Filtered & & \\
\hline & $\begin{array}{l}\mathrm{M} 2 \mathrm{M} 2-7 \\
\left(2.4^{\circ}-0^{\circ} \mathrm{S}\right)\end{array}$ & $844-1025$ & $4.71-4.91$ & 4.95 & 6.25 & 12.83 & $\begin{array}{c}6(890), \\
5.2(940)\end{array}$ & $\begin{array}{c}11(924), \\
11.5(1025)\end{array}$ & & $\begin{array}{l}\text { Crest }\left(2.3^{\circ} \mathrm{S}\right) \text {, } \\
\text { Trough } \\
\left(1.4^{\circ} \mathrm{S}\right), \\
\text { Crest }\left(0.6^{\circ} \mathrm{S}\right)\end{array}$ \\
\hline $\begin{array}{l}\text { M3M3-A } \\
\left(18^{\circ}-\right. \\
13.91^{\circ} \mathrm{S}\end{array}$ & $\begin{array}{l}\text { M3M3-A1 } \\
\left(18^{\circ}-16.9^{\circ} \mathrm{S}\right)\end{array}$ & $0-113$ & $4.75-5.27$ & 5.1 & 5.96 & 9.87 & $\begin{array}{c}6.5(30), \\
6(50), \\
7(70), \\
5.8(90)\end{array}$ & $\begin{array}{c}11.5(50), \\
11.2(70), \\
10(90), \\
8.8(110)\end{array}$ & $\begin{array}{l}\text { Crest } \\
\left(17.3^{\circ} \mathrm{S}\right)\end{array}$ & \\
\hline \multirow[t]{4}{*}{$\begin{array}{l}\text { S along } \\
\left.\sim 79^{\circ} \mathrm{E}\right)\end{array}$} & $\begin{array}{l}\mathrm{M} 3 \mathrm{M} 3-\mathrm{A} 2 \\
\left(16.2^{\circ}-\right. \\
\left.17.1^{\circ} \mathrm{S}\right)\end{array}$ & $100-200$ & $4.57-5.27$ & 4.99 & 5.64 & 10.72 & $\begin{array}{l}5.8(110), \\
6.6(120), \\
5.8(170)\end{array}$ & $\begin{array}{l}10.8(150) \\
11.6(190)\end{array}$ & $\begin{array}{l}\text { Trough } \\
\left(16.7^{\circ} \mathrm{S}\right)\end{array}$ & \\
\hline & $\begin{array}{l}\text { M3M3-A3 } \\
\left(17.1^{\circ}-\right. \\
\left.15.5^{\circ} \mathrm{S}\right)\end{array}$ & $177-275$ & $4.82-5.07$ & 5.02 & 5.64 & 12.27 & $\begin{array}{l}5.4(200) \\
5.2(270)\end{array}$ & $\begin{array}{c}12(210), \\
12.4(240) \\
12(280)\end{array}$ & & \\
\hline & $\begin{array}{l}\mathrm{M} 3 \mathrm{M} 3-\mathrm{A} 4 \\
\left(15.6^{\circ}-\right. \\
\left.15.2^{\circ} \mathrm{S}\right)\end{array}$ & $267-313$ & $4.72-4.96$ & 4.89 & 5.05 & 12.8 & $7.2(290)$ & $11.8(310)$ & $\begin{array}{l}\text { Crest } \\
\left(15.26^{\circ} \mathrm{S}\right)\end{array}$ & \\
\hline & $\begin{array}{l}\text { M3M3-A5 } \\
\left(15.2^{\circ}-\right. \\
\left.13.91^{\circ} \mathrm{S}\right)\end{array}$ & $306-450$ & $4.85-5.01$ & 5.00 & 6.5 & 11.59 & $\begin{array}{c}6(330), \\
6.8(370), \\
5(415), \\
7(430)\end{array}$ & $\begin{array}{l}10.8(340), \\
11.5(385), \\
10.8(406), \\
10.2(450)\end{array}$ & $\begin{array}{l}\text { Trough } \\
\left(14.08^{\circ} \mathrm{S}\right)\end{array}$ & \\
\hline \multirow[t]{3}{*}{$\begin{array}{l}\text { M3M3-B } \\
\left(12.91^{\circ}-\right. \\
9^{\circ} \mathrm{S} \text { along } \\
\left.\sim 79^{\circ} \mathrm{E}\right)\end{array}$} & $\begin{array}{l}\text { M3M3-B1 } \\
\left(12.9^{\circ}-\right. \\
\left.11.61^{\circ} \mathrm{S}\right)\end{array}$ & $0-150$ & $4.76-4.98$ & 4.95 & 5.69 & 11.43 & $\begin{array}{l}5.3(20), \\
6.4(65), \\
5.2(100) \\
5.2(115)\end{array}$ & $11(56)$ & & \\
\hline & $\begin{array}{l}\text { M3M3-B2 } \\
\left(11.91^{\circ}-\right. \\
\left.10.73^{\circ} \mathrm{S}\right)\end{array}$ & $115-250$ & $4.86-5.05$ & 5.08 & 5.45 & 12.23 & $\begin{array}{l}7.2(140), \\
5.4(170), \\
5.2(210)\end{array}$ & $\begin{array}{c}10(160), \\
10.4(196)\end{array}$ & & \\
\hline & $\begin{array}{l}\text { M3M3-B3 } \\
\left(10.95^{\circ}-9^{\circ} \mathrm{S}\right)\end{array}$ & $221-442$ & $5.15-5.42$ & 5.43 & 5.2 & 10.26 & $\begin{array}{l}5.6(410), \\
5.4(420)\end{array}$ & $\begin{array}{l}11.2(340), \\
10.8(380), \\
10.3(410), \\
10.2(440)\end{array}$ & $\begin{array}{l}\text { Crest } \\
\left(9.5^{\circ} \mathrm{S}\right)\end{array}$ & \\
\hline \multirow{6}{*}{$\begin{array}{l}\text { M4M4 } \\
\left(18^{\circ}-\right. \\
10^{\circ} \mathrm{S} \\
\text { along } \\
\left.\sim 80^{\circ} \mathrm{E}\right)\end{array}$} & $\begin{array}{l}\text { M4M4-1 } \\
\left(18^{\circ}-\right. \\
\left.16.56^{\circ} \mathrm{S}\right)\end{array}$ & $0-156$ & $4.9-5.28$ & 5.09 & 5.75 & 11.98 & $\begin{array}{l}5.8(20), \\
6.4(60), \\
5.5(150)\end{array}$ & $\begin{array}{c}11(0) \\
12.6(70), \\
12(130)\end{array}$ & & \\
\hline & $\begin{array}{l}\text { M4M4-2 } \\
\left(16.7^{\circ}-\right. \\
\left.15.3^{\circ} \mathrm{S}\right)\end{array}$ & $146-304$ & $4.8-5.14$ & 4.97 & 5.36 & 10.62 & $\begin{array}{c}5.6(160), \\
6(190), \\
6.2(240), \\
6(280)\end{array}$ & $\begin{array}{l}10.8(176), \\
10.4(226), \\
10.8(266)\end{array}$ & $\begin{array}{l}\text { Crest } \\
\left(12.8^{\circ} \mathrm{S}\right)\end{array}$ & \\
\hline & $\begin{array}{l}\text { M4M4-3 } \\
\left(15.54^{\circ}-\right. \\
\left.14.9^{\circ} \mathrm{S}\right)\end{array}$ & $272-342$ & $4.95-5.13$ & 5.12 & 5.7 & 11.7 & $6.6(340)$ & $\begin{array}{c}10.9(312) \\
11(342)\end{array}$ & $\begin{array}{l}\text { Trough } \\
\left(14.9^{\circ} \mathrm{S}\right)\end{array}$ & \\
\hline & $\begin{array}{l}\text { M4M4-4 } \\
\left(14.89^{\circ}-\right. \\
\left.13.6^{\circ} \mathrm{S}\right)\end{array}$ & $339-492$ & $4.84-5.1$ & 5.05 & 5.5 & 11.95 & $\begin{array}{l}5.4(360), \\
6.8(400), \\
6.3(430), \\
5.6(490)\end{array}$ & $\begin{array}{l}12(410), \\
13(450)\end{array}$ & & \\
\hline & $\begin{array}{l}\text { M4M4-5 } \\
\left(13.7^{\circ}-\right. \\
\left.12.3^{\circ} \mathrm{S}\right)\end{array}$ & $453-637$ & $3.84-5.17$ & 4.6 & 5.73 & 13.2 & $\begin{array}{c}6(550), \\
5.6(580), \\
5.2(620)\end{array}$ & $\begin{array}{l}12.5(525), \\
12.6(575)\end{array}$ & $\begin{array}{l}\text { Crest } \\
\left(12.8^{\circ} \mathrm{S}\right)\end{array}$ & \\
\hline & $\begin{array}{l}\text { M4M4-6 } \\
\left(12.31^{\circ}-\right. \\
\left.10.4^{\circ} \mathrm{S}\right)\end{array}$ & $630-843$ & $4.32-5.4$ & 4.97 & 6.2 & 11.5 & $\begin{array}{l}6.8(670), \\
6.5(740), \\
5.4(780), \\
5.0(840)\end{array}$ & $\begin{array}{c}12.8(660), \\
11(750), \\
11.5(780), \\
11(843)\end{array}$ & $\begin{array}{l}\text { Trough } \\
\left(11.5^{\circ} \mathrm{S}\right)\end{array}$ & \\
\hline
\end{tabular}


Table 1. (Contd)

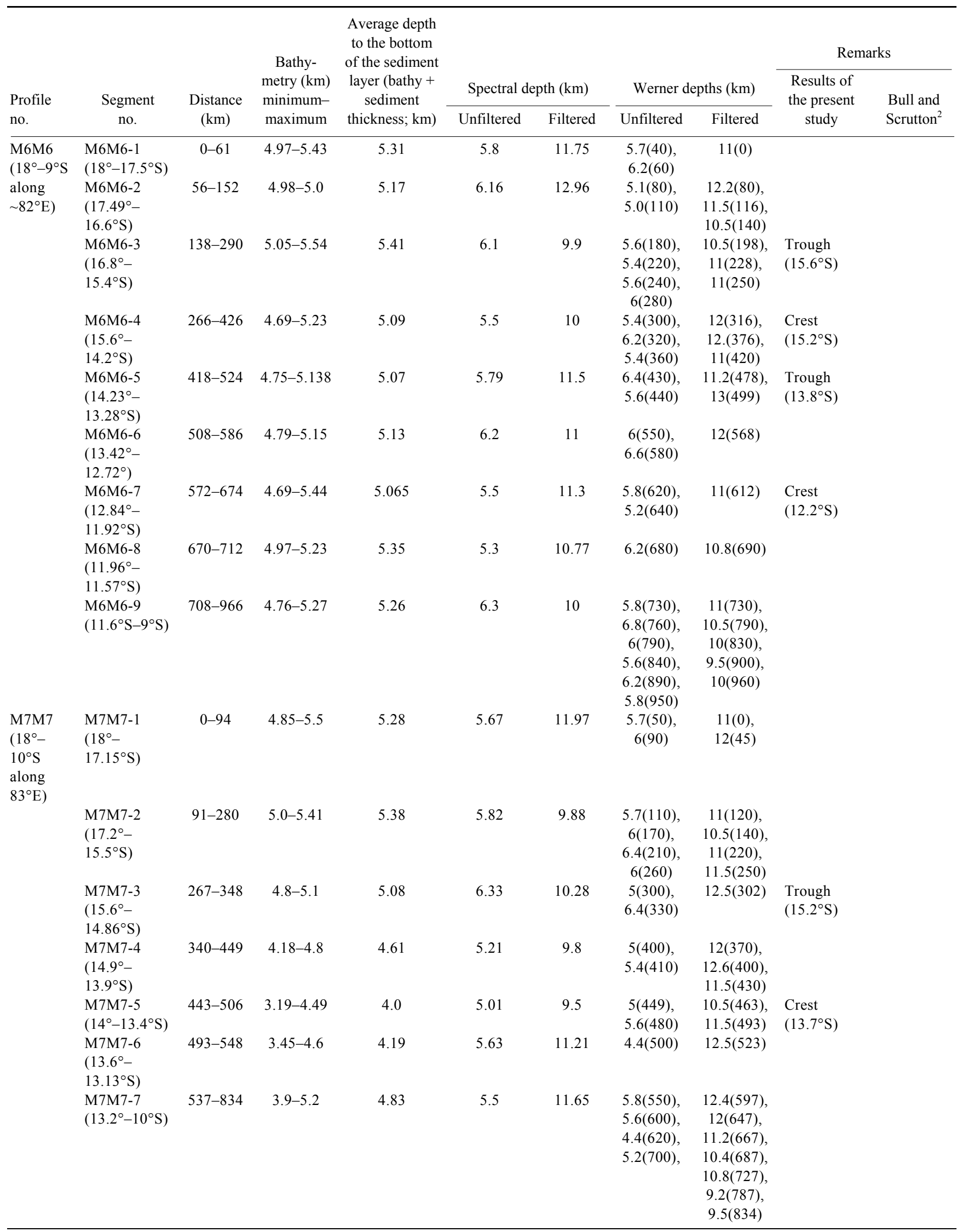


RESEARCH ARTICLES

Table 1. (Contd)

\begin{tabular}{|c|c|c|c|c|c|c|c|c|c|c|}
\hline \multirow{3}{*}{$\begin{array}{l}\text { Profile } \\
\text { no. }\end{array}$} & \multirow{3}{*}{$\begin{array}{l}\text { Segment } \\
\text { no. }\end{array}$} & \multirow{3}{*}{$\begin{array}{c}\text { Distance } \\
(\mathrm{km})\end{array}$} & \multirow{3}{*}{$\begin{array}{l}\text { Bathy- } \\
\text { metry }(\mathrm{km}) \\
\text { minimum- } \\
\text { maximum }\end{array}$} & \multirow{3}{*}{$\begin{array}{l}\text { Average depth } \\
\text { to the bottom } \\
\text { of the sediment } \\
\text { layer (bathy }+ \\
\text { sediment } \\
\text { thickness; km) }\end{array}$} & \multirow{2}{*}{\multicolumn{2}{|c|}{ Spectral depth (km) }} & \multirow{2}{*}{\multicolumn{2}{|c|}{ Werner depths (km) }} & \multicolumn{2}{|c|}{ Remarks } \\
\hline & & & & & & & & & & \\
\hline & & & & & Unfiltered & Filtered & Unfiltered & Filtered & study & Scrutton $^{2}$ \\
\hline \multirow[t]{8}{*}{$\begin{array}{l}\text { M8M8 } \\
\left(18^{\circ}-8^{\circ} \mathrm{S}\right. \\
\text { along } \\
\left.\sim 84^{\circ} \mathrm{E}\right)\end{array}$} & $\begin{array}{l}\text { M8M8-1 } \\
\left(18^{\circ}-16.1^{\circ} \mathrm{S}\right)\end{array}$ & $0-215$ & $4.99-5.36$ & 5.33 & 6.18 & 10.58 & $\begin{array}{c}5.6(40), \\
5.8(80), \\
5.6(100), \\
6.8(130), \\
5.8(175)\end{array}$ & $\begin{array}{c}11(0), \\
10(35), \\
11.5(70), \\
11.6(140), \\
11.8(160), \\
11(180)\end{array}$ & & \\
\hline & $\begin{array}{l}\text { M8M8-2 } \\
\left(16.5^{\circ}-\right. \\
\left.14.9^{\circ} \mathrm{S}\right)\end{array}$ & $168-338$ & $4.57-5.38$ & 5.125 & 6.67 & 12.84 & $\begin{array}{c}6.8(210), \\
6.2(230), \\
5.1(270), \\
6(310)\end{array}$ & $\begin{array}{c}11.5(203) \\
12(233) \\
13(245)\end{array}$ & $\begin{array}{l}\text { Trough } \\
\left(15.7^{\circ} \mathrm{S}\right)\end{array}$ & \\
\hline & $\begin{array}{l}\text { M8M8-3 } \\
\left(15.4^{\circ}-\right. \\
\left.13.9^{\circ} \mathrm{S}\right)\end{array}$ & $289-456$ & $3.5-4.91$ & 4.36 & 5.6 & 10.46 & $\begin{array}{l}6(335), \\
4.4(366), \\
5.6(370), \\
6.2(390)\end{array}$ & $\begin{array}{l}12(334), \\
11(389), \\
11.5(410)\end{array}$ & & \\
\hline & $\begin{array}{l}\text { M8M8-4 } \\
\left(14.2^{\circ}-\right. \\
\left.12.4^{\circ} \mathrm{S}\right)\end{array}$ & $426-623$ & $4.13-5.2$ & 4.84 & 5.09 & 12.43 & $\begin{array}{l}6.2(460), \\
4.8(540), \\
5.8(545), \\
6.2(580)\end{array}$ & $\begin{array}{c}11.5(526), \\
12(546), \\
12.5(576)\end{array}$ & $\begin{array}{l}\text { Crest } \\
\left(13.5^{\circ} \mathrm{S}\right)\end{array}$ & \\
\hline & $\begin{array}{l}\text { M8M8-5 } \\
\left(12.6^{\circ}-\right. \\
\left.12.2^{\circ} \mathrm{S}\right)\end{array}$ & $599-649$ & $4.67-5.24$ & 5.11 & 5.2 & 11.7 & $6(640)$ & $13(620)$ & $\begin{array}{l}\text { Trough } \\
\left(12.6^{\circ} \mathrm{S}\right)\end{array}$ & \\
\hline & $\begin{array}{l}\text { M8M8-6 } \\
\left(12.25^{\circ}-\right. \\
\left.11.1^{\circ} \mathrm{S}\right)\end{array}$ & $639-767$ & $3.3-5.01$ & 4.37 & 5 & 10.59 & $\begin{array}{c}5.2(720) \\
6(760)\end{array}$ & 11(709) & & \\
\hline & $\begin{array}{l}\text { M8M8-7 } \\
\left(11.2^{\circ}-\right. \\
\left.9.8^{\circ} \mathrm{S}\right)\end{array}$ & $759-906$ & $4.53-5.34$ & 5.26 & 5.2 & 11.52 & $\begin{array}{l}5.4(830), \\
5.2(850), \\
5.7(880)\end{array}$ & $\begin{array}{l}10.5(799), \\
10.5(830), \\
10.5(870)\end{array}$ & $\begin{array}{l}\text { Crest } \\
\left(10.3^{\circ} \mathrm{S}\right)\end{array}$ & \\
\hline & $\begin{array}{l}\text { M8M8-8 } \\
\left(9.89^{\circ} \mathrm{S}-8^{\circ} \mathrm{S}\right)\end{array}$ & $\begin{array}{l}901- \\
1105\end{array}$ & $4.64-5.29$ & 5.26 & 5.99 & 10.79 & $\begin{array}{c}6(940), \\
6.4(980), \\
5.8(1050), \\
5.4(1100)\end{array}$ & $\begin{array}{c}12(930), \\
11.5(970), \\
11(990), \\
10(1105)\end{array}$ & $\begin{array}{l}\text { Trough } \\
\left(8.98^{\circ} \mathrm{S}\right)\end{array}$ & \\
\hline \multirow[t]{5}{*}{$\begin{array}{l}\text { M9M9 } \\
\left(77^{\circ} \mathrm{E},\right. \\
15^{\circ} 30^{\circ}- \\
79^{\circ} \mathrm{E}, \\
\left.6^{\circ} \mathrm{N}\right)\end{array}$} & $\begin{array}{l}\text { M9M9-1 } \\
\left(15.5^{\circ} \mathrm{S},\right. \\
76.9^{\circ} \mathrm{E}- \\
13.9^{\circ} \mathrm{S}, \\
\left.77.1^{\circ} \mathrm{E}\right)\end{array}$ & $0-181$ & $4.9-5.4$ & 5.22 & 5.48 & 9.98 & $\begin{array}{c}5(30) \\
7.2(90) \\
5.2(110)\end{array}$ & $\begin{array}{c}10(0), \\
10.4(15), \\
10.8(50), \\
11.2(80), \\
11.5(100), \\
10.8(160)\end{array}$ & & \\
\hline & $\begin{array}{l}\text { M9M9-2 } \\
\left(13.9^{\circ} \mathrm{S},\right. \\
77.1^{\circ}- \\
13.5^{\circ} \mathrm{S}, \\
\left.77.2^{\circ} \mathrm{E}\right)\end{array}$ & $169-228$ & $5.1-5.5$ & 5.39 & 5.82 & 10.55 & $6(170)$ & $10.4(220)$ & & \\
\hline & $\begin{array}{l}\text { M9M9-3 } \\
\left(13.5^{\circ} \mathrm{S},\right. \\
71.16^{\circ} \mathrm{E}- \\
11.4^{\circ} \mathrm{S}, \\
\left.77.3^{\circ} \mathrm{E}\right)\end{array}$ & $224-459$ & $5.09-5.67$ & 5.47 & 5.64 & 10.64 & $\begin{array}{c}6(250), \\
6.4(330), \\
5.8(430)\end{array}$ & & & \\
\hline & $\begin{array}{l}\text { M9M9-4 } \\
\left(11.5^{\circ} \mathrm{S},\right. \\
77.3^{\circ} \mathrm{E}- \\
10.5^{\circ} \mathrm{S}, \\
\left.77.4^{\circ} \mathrm{E}\right)\end{array}$ & $450-561$ & $5.28-5.5$ & 5.5 & 5.9 & 10.21 & $\begin{array}{l}6.8(510), \\
5.4(520), \\
6.0(550)\end{array}$ & $12.4(540)$ & $\begin{array}{l}\text { Trough } \\
\left(11.1^{\circ} \mathrm{S},\right. \\
\left.77.32^{\circ} \mathrm{E}\right)\end{array}$ & \\
\hline & $\begin{array}{l}\text { M9M9-5 } \\
\left(10.5^{\circ} \mathrm{S},\right. \\
77.4^{\circ} \mathrm{E}- \\
8.6^{\circ} \mathrm{S}, \\
\left.77.57^{\circ} \mathrm{E}\right)\end{array}$ & $552-769$ & $5.3-5.6$ & 5.6 & 5.3 & 9.17 & $\begin{array}{l}5.2(550), \\
5.6(630), \\
6.2(650), \\
5.4(670), \\
5.5(690)\end{array}$ & $\begin{array}{l}11.6(620), \\
12.4(760)\end{array}$ & $\begin{array}{l}\text { Crest } \\
\left(9.5^{\circ} \mathrm{S},\right. \\
\left.77.4^{\circ} \mathrm{E}\right)\end{array}$ & \\
\hline
\end{tabular}


Table 1. (Contd)

\begin{tabular}{|c|c|c|c|c|c|c|c|c|c|c|}
\hline \multirow{3}{*}{$\begin{array}{l}\text { Profile } \\
\text { no. }\end{array}$} & \multirow{3}{*}{$\begin{array}{l}\text { Segment } \\
\text { no. }\end{array}$} & \multirow{3}{*}{$\begin{array}{l}\text { Distance } \\
(\mathrm{km})\end{array}$} & \multirow{3}{*}{$\begin{array}{l}\text { Bathy- } \\
\text { metry }(\mathrm{km}) \\
\text { minimum- } \\
\text { maximum }\end{array}$} & \multirow{3}{*}{$\begin{array}{l}\text { Average depth } \\
\text { to the bottom } \\
\text { of the sediment } \\
\text { layer (bathy + } \\
\text { sediment } \\
\text { thickness; km) }\end{array}$} & \multirow{2}{*}{\multicolumn{2}{|c|}{ Spectral depth $(\mathrm{km})$}} & \multirow{2}{*}{\multicolumn{2}{|c|}{ Werner depths $(\mathrm{km})$}} & \multicolumn{2}{|c|}{ Remarks } \\
\hline & & & & & & & & & \multirow{2}{*}{$\begin{array}{c}\text { Results of } \\
\text { the present } \\
\text { study }\end{array}$} & \multirow{2}{*}{$\begin{array}{l}\text { Bull and } \\
\text { Scrutton }^{2}\end{array}$} \\
\hline & & & & & Unfiltered & Filtered & Unfiltered & Filtered & & \\
\hline & $\begin{array}{l}\text { M9M9-6 } \\
\left(8.53^{\circ} \mathrm{S},\right. \\
77.6^{\circ} \mathrm{E}- \\
7.1^{\circ} \mathrm{S}, \\
\left.77.7^{\circ} \mathrm{E}\right)\end{array}$ & $760-936$ & $5.0-5.67$ & 5.55 & 5.54 & 10.13 & $\begin{array}{l}5.8(770), \\
6.4(820), \\
5.2(870)\end{array}$ & $\begin{array}{l}12.4(780), \\
11.6(830), \\
11.4(880)\end{array}$ & & \\
\hline & $\begin{array}{l}\text { M9M9-7 } \\
\left(7.4^{\circ} \mathrm{S},\right. \\
77.7 \mathrm{E}, \\
6.52^{\circ} \mathrm{S}, \\
\left.77.8^{\circ} \mathrm{E}\right)\end{array}$ & $902-1000$ & $5.0-5.3$ & 5.4 & 5.67 & 12.4 & $\begin{array}{c}5.45(940) \\
5.3(950)\end{array}$ & $\begin{array}{c}10.4(930) \\
13(957)\end{array}$ & & \\
\hline & $\begin{array}{l}\text { M9M9-8 } \\
\left(6.6^{\circ} \mathrm{S},\right. \\
77.8^{\circ} \mathrm{E}- \\
\left.4.8^{\circ} \mathrm{S}, 7.9^{\circ} \mathrm{E}\right)\end{array}$ & $986-1185$ & $4.89-5.22$ & 5.31 & 6.62 & 11.32 & $\begin{array}{c}5.6(1000), \\
6(1040)\end{array}$ & $\begin{array}{l}12.8(1000), \\
12.4(1050), \\
11.4(1160)\end{array}$ & $\begin{array}{l}\text { Trough } \\
\left(5.87^{\circ} \mathrm{S},\right. \\
\left.77.85^{\circ} \mathrm{E}\right)\end{array}$ & \\
\hline & $\begin{array}{l}\text { M9M9-9 } \\
\left(5.4^{\circ} \mathrm{S}\right. \\
77.88^{\circ} \mathrm{E}- \\
\left.3.7^{\circ} \mathrm{S}, 78^{\circ} \mathrm{E}\right)\end{array}$ & $\begin{array}{c}1123- \\
1315\end{array}$ & $4.66-5.07$ & 5.12 & 5.5 & 10.31 & $\begin{array}{c}4.8(1250) \\
5.2(1290)\end{array}$ & $\begin{array}{c}12(1240), \\
12(1260), \\
11.8(1280)\end{array}$ & $\begin{array}{l}\text { Crest } \\
\left(5.85^{\circ} \mathrm{S},\right. \\
\left.77.8^{\circ} \mathrm{E}\right)\end{array}$ & \\
\hline & $\begin{array}{l}\text { M9M9-10 } \\
\left(4.08^{\circ} \mathrm{S},\right. \\
78^{\circ} \mathrm{E}-0.2^{\circ} \mathrm{S}, \\
\left.78.4^{\circ} \mathrm{E}\right)\end{array}$ & $\begin{array}{c}1270- \\
1701\end{array}$ & $4.74-5.08$ & 5.59 & 5.98 & 10.64 & $\begin{array}{l}6.4(1360), \\
5.8(1440), \\
5.8(1490), \\
5.8(1510), \\
5.6(1580), \\
5.8(1630)\end{array}$ & $\begin{array}{c}10.7(1430), \\
11(1490), \\
11.2(1580), \\
12.2(1650)\end{array}$ & $\begin{array}{l}\text { Trough } \\
\left(2.87^{\circ} \mathrm{S},\right. \\
\left.78.65^{\circ} \mathrm{E}\right)\end{array}$ & \\
\hline & $\begin{array}{l}\text { M9M9-11 } \\
\left(0.58^{\circ} \mathrm{S},\right. \\
78.4^{\circ} \mathrm{E}- \\
1.61^{\circ} \mathrm{N}, \\
\left.78.6^{\circ} \mathrm{E}\right)\end{array}$ & $\begin{array}{c}1629- \\
1902\end{array}$ & $4.58-4.8$ & 5.38 & 5.25 & 10.95 & $\begin{array}{l}6(1700), \\
4.6(1730), \\
5.2(1770), \\
5.6(1810), \\
5.7(1870), \\
5.4(1890)\end{array}$ & $\begin{array}{c}13.6(1740) \\
13.2(1880)\end{array}$ & & \\
\hline & $\begin{array}{l}\text { M9M9-12 } \\
0.69^{\circ} \mathrm{N}, \\
78.6^{\circ} \mathrm{E}- \\
2.6^{\circ} \mathrm{N}, \\
\left.78.7^{\circ} \mathrm{E}\right)\end{array}$ & $\begin{array}{c}1800- \\
2016\end{array}$ & $3.45-4.32$ & 4.24 & 5.44 & 11.79 & $\begin{array}{c}5.2(1910) \\
5.4(2010)\end{array}$ & $\begin{array}{c}12(1920) \\
11.5(1980)\end{array}$ & & \\
\hline & $\begin{array}{l}\text { M9M9-13 } \\
\left(2.6^{\circ} \mathrm{N},\right. \\
78.7^{\circ} \mathrm{E}- \\
3.7^{\circ} \mathrm{N}, \\
78.8^{\circ} \mathrm{E}\end{array}$ & $\begin{array}{c}2008- \\
2138\end{array}$ & $3.08-4.32$ & 3.94 & 4.28 & 11.3 & $4.4(2130)$ & $\begin{array}{c}10.8(2030) \\
12(2134)\end{array}$ & $\begin{array}{l}\text { Crest } \\
\left(3.07^{\circ} \mathrm{N},\right. \\
\left.78.7^{\circ} \mathrm{E}\right)\end{array}$ & \\
\hline & $\begin{array}{l}\text { M9M9-14 } \\
\left(3.49^{\circ} \mathrm{N},\right. \\
78.78^{\circ} \mathrm{E}- \\
5.74^{\circ} \mathrm{N} \\
78.98^{\circ} \mathrm{E}\end{array}$ & $\begin{array}{c}2114- \\
2363\end{array}$ & $3.40-4.4$ & 4.62 & 5.6 & 11.8 & $\begin{array}{l}4.6(2180), \\
5.2(2230), \\
6.4(2250), \\
6(2290), \\
6.8(2300), \\
5.2(2340)\end{array}$ & $\begin{array}{l}9.6(2240), \\
11.2(2363)\end{array}$ & & \\
\hline \multirow[t]{2}{*}{$\begin{array}{l}\mathrm{M} 10 \mathrm{M} 10 \\
\left(7.5^{\circ} \mathrm{S},\right. \\
79^{\circ} \mathrm{E}- \\
3.5^{\circ} \mathrm{N}, \\
\left.76^{\circ} \mathrm{E}\right)\end{array}$} & $\begin{array}{l}\mathrm{M} 10 \mathrm{M} 10-1 \\
\left(7.5^{\circ} \mathrm{S},\right. \\
79^{\circ} \mathrm{E}-6.4^{\circ} \mathrm{S}, \\
\left.78.6^{\circ} \mathrm{E}\right)\end{array}$ & $0-175$ & $4.79-5.09$ & 5.39 & 6.08 & 9.79 & $\begin{array}{c}6(40), \\
5.6(60), \\
6(130), \\
5.4(160)\end{array}$ & $\begin{array}{c}10(0), \\
10.4(20), \\
10.5(60), \\
10.4(90), \\
10.6(120)\end{array}$ & & \\
\hline & $\begin{array}{l}\mathrm{M} 10 \mathrm{M} 10-2 \\
\left(6.5^{\circ} \mathrm{S},\right. \\
78.7^{\circ} \mathrm{E} \\
5.3^{\circ} \mathrm{S} \\
\left.78.4^{\circ} \mathrm{E}\right)\end{array}$ & $157-94$ & $4.8-5.09$ & 5.39 & 6 & 8.47 & $\begin{array}{c}6.0(190), \\
5.2(210), \\
7(240), \\
5.6(280)\end{array}$ & $\begin{array}{c}10.4(160) \\
10.8(180) \\
11(280)\end{array}$ & & \\
\hline
\end{tabular}


RESEARCH ARTICLES

Table 1. (Contd)

\begin{tabular}{|c|c|c|c|c|c|c|c|c|c|c|}
\hline \multirow{3}{*}{$\begin{array}{l}\text { Profile } \\
\text { no. }\end{array}$} & \multirow{3}{*}{$\begin{array}{c}\text { Segment } \\
\text { no. }\end{array}$} & \multirow{3}{*}{$\begin{array}{c}\text { Distance } \\
(\mathrm{km})\end{array}$} & \multirow{3}{*}{$\begin{array}{l}\text { Bathy- } \\
\text { metry }(\mathrm{km}) \\
\text { minimum- } \\
\text { maximum }\end{array}$} & \multirow{3}{*}{$\begin{array}{l}\text { Average depth } \\
\text { to the bottom } \\
\text { of the sediment } \\
\text { layer (bathy + } \\
\text { sediment } \\
\text { thickness; km) }\end{array}$} & \multirow{2}{*}{\multicolumn{2}{|c|}{ Spectral depth $(\mathrm{km})$}} & \multirow{2}{*}{\multicolumn{2}{|c|}{ Werner depths $(\mathrm{km})$}} & \multicolumn{2}{|c|}{ Remarks } \\
\hline & & & & & & & & & \multirow{2}{*}{$\begin{array}{c}\text { Results of } \\
\text { the present } \\
\text { study }\end{array}$} & \multirow{2}{*}{$\begin{array}{l}\text { Bull and } \\
\text { Scrutton }^{2}\end{array}$} \\
\hline & & & & & Unfiltered & Filtered & Unfiltered & Filtered & & \\
\hline & $\begin{array}{l}\mathrm{M} 10 \mathrm{M} 10-3 \\
\left(5.4^{\circ} \mathrm{S},\right. \\
78.4^{\circ} \mathrm{E}- \\
4.27^{\circ} \mathrm{S} \\
\left.78.1^{\circ} \mathrm{E}\right)\end{array}$ & $285-414$ & $4.87-4.99$ & 5.15 & 5.8 & 11.7 & $\begin{array}{c}6.2(300) \\
6(350) \\
7(400)\end{array}$ & $11.2(350)$ & & \\
\hline & $\begin{array}{l}\mathrm{M} 10 \mathrm{M} 10-4 \\
\left(4.4^{\circ} \mathrm{S},\right. \\
78.2^{\circ} \mathrm{E}- \\
2.38^{\circ} \mathrm{S} \\
\left.77.7^{\circ} \mathrm{E}\right)\end{array}$ & $403-629$ & $4.5-4.8$ & 4.96 & 5.65 & 12.54 & $\begin{array}{c}5.4(430), \\
6.8(480), \\
5.4(530), \\
6(550), \\
5.5(570), \\
7(580), \\
5.8(620)\end{array}$ & $\begin{array}{c}11.4(423), \\
10.6(460), \\
9.8(480), \\
11.2(523), \\
11.8(540), \\
10(610)\end{array}$ & $\begin{array}{l}\text { Trough } \\
\left(3.59^{\circ} \mathrm{S},\right. \\
\left.77.87^{\circ} \mathrm{E}\right)\end{array}$ & \\
\hline & $\begin{array}{l}\mathrm{M} 10 \mathrm{M} 10-5 \\
\left(2.49^{\circ} \mathrm{S},\right. \\
77.7^{\circ} \mathrm{E}- \\
0.9^{\circ} \mathrm{S}, \\
\left.77.3^{\circ} \mathrm{E}\right)\end{array}$ & $615-795$ & $3.4-4.22$ & 4.18 & 5.6 & 10.53 & $\begin{array}{l}6.2(687), \\
6(7578), \\
5.9(777)\end{array}$ & $\begin{array}{l}11(670) \\
10(765)\end{array}$ & & \\
\hline & $\begin{array}{l}\mathrm{M} 10 \mathrm{M} 10-6 \\
\left(1.05^{\circ} \mathrm{S}\right. \\
77.4^{\circ} \mathrm{E}- \\
0.74^{\circ} \mathrm{N} \\
\left.76.9^{\circ} \mathrm{E}\right)\end{array}$ & $780-983$ & $3.2-4.22$ & 4.0 & 4.52 & 9.28 & $\begin{array}{c}6.8(827), \\
6(857), \\
7(877), \\
6.3(927), \\
6.2(977)\end{array}$ & $\begin{array}{l}12.2(800), \\
11.4(820), \\
12.4(870), \\
10.2(920), \\
10.2(960)\end{array}$ & $\begin{array}{l}\text { Crest } \\
\left(0.5^{\circ} \mathrm{S},\right. \\
\left.76.8^{\circ} \mathrm{E}\right)\end{array}$ & \\
\hline & $\begin{array}{l}\mathrm{M} 10 \mathrm{M} 10-7 \\
\left(0.7^{\circ} \mathrm{N},\right. \\
76.9^{\circ} \mathrm{E}- \\
\left.3.5^{\circ} \mathrm{N}, 76^{\circ} \mathrm{E}\right)\end{array}$ & $977-1288$ & $2.06-3.37$ & 2.98 & 3.45 & 10.87 & $\begin{array}{l}4.4(997), \\
5.6(1032), \\
0.2(1117), \\
5.8(1167), \\
5.4(1237), \\
5.2(1257), \\
4.4(1277), \\
3.2(1288)\end{array}$ & $\begin{array}{c}10(987), \\
10.6(1029), \\
10.4(1080) \\
11.2(1090), \\
11.6(1177), \\
11.2(1288)\end{array}$ & & \\
\hline
\end{tabular}

Values within brackets indicate distances in the segment where depths were noted.

The inferences of troughs and crests of Bull and Scrutton $^{2}$ in the study area are shown in Table 1 along with those of our findings. Numerous large (height $>1000 \mathrm{~m}$ ) and small (height $<1000 \mathrm{~m}$ ) sea mounts were identified in the study $\operatorname{area}^{28}$.

\section{Results and discussion}

\section{M1M1 profile}

This profile is about $1438 \mathrm{~km}$ long and is in between $9^{\circ} \mathrm{S}$ and $4^{\circ} \mathrm{N}$ lat. and along $80^{\circ} \mathrm{E}$ long. The profile runs adjacent and parallel to $80^{\circ} \mathrm{E} \mathrm{FZ} \mathrm{(Figure} \mathrm{1).} \mathrm{Following}$ the procedure mentioned above, the profile was divided into 11 segments (Table 1). The spectral depth to the top and bottom of the oceanic crust was determined from the unfiltered and filtered magnetic anomalies. The same unfiltered and filtered magnetic anomaly segments were subjected to Werner deconvolution technique to get the top and bottom depths of the oceanic crust respectively. Table 1 shows all the depth values. The spectral depths to the marine magnetic basement were in the range 5.6$6.42 \mathrm{~km}$ and deeper magnetic marker (Curie isotherm) in the depth range $9.1-12.15 \mathrm{~km}$. The depths obtained from Werner deconvolution to shallow and deeper magnetic markers were in the range $5.2-7.2 \mathrm{~km}$ and $9.6-14.4 \mathrm{~km}$ respectively.

The variations in the top and bottom depths of both spectral and Werner deconvolution techniques indicate undulations of the crust. From these depths a tentative crustal model was prepared.

From the tentative model (Figure 2), uplifts (crests) at $8.35^{\circ} \mathrm{S}$ and troughs at $6.8^{\circ} \mathrm{S}$ and $1.44^{\circ} \mathrm{S}$ were observed. These crests and troughs coincided well with those inferred by Bull and Scrutton ${ }^{2}$ (Table 1). The undulating crust model indicates deformation. There was a good agreement between the depths of spectral and Werner deconvolution techniques (Figure 2). 


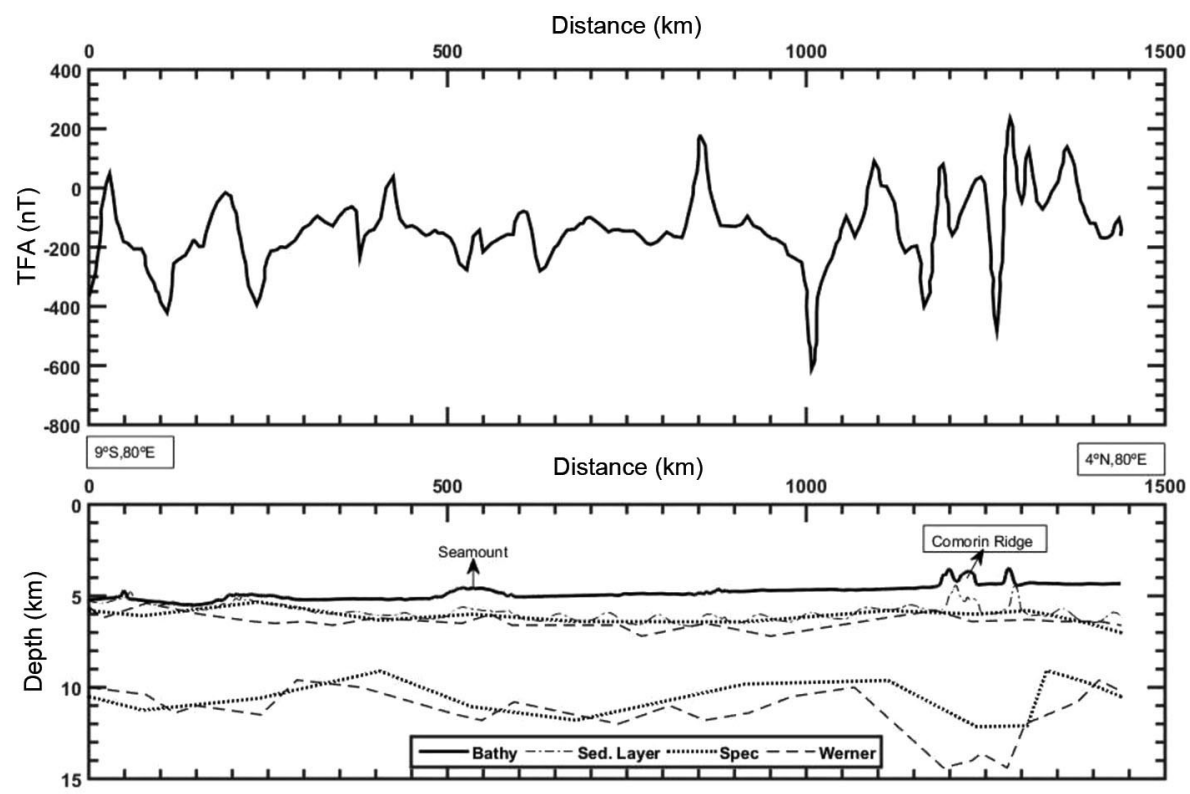

Figure 2. (Top) Total field magnetic anomaly (TFA) along profile M1M1. (Bottom) Tentative crustal model derived from spectral and Werner deconvolution analysis along with bathymetry and sedimentary layers.

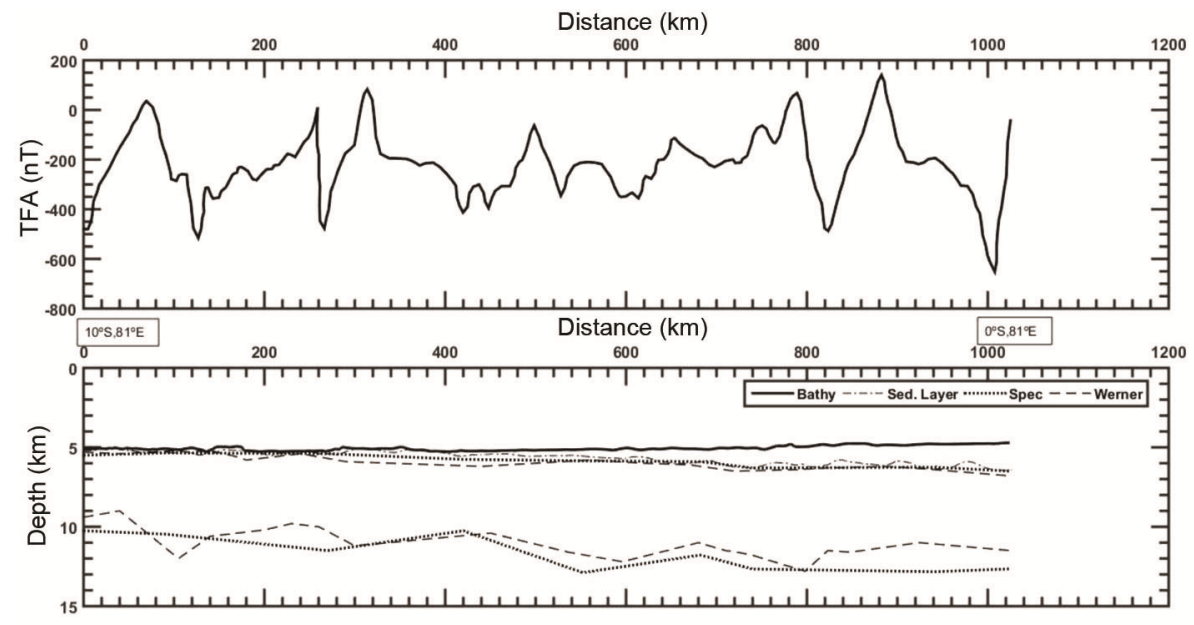

Figure 3. (Top) TFA along profile M2M2. (Bottom) Tentative crustal model derived from spectral and Werner deconvolution analysis along with bathymetry and sedimentary layers.

The bathymetry and sediment layers showed an increase at $500-550 \mathrm{~km}$ and $\sim 1200-1300 \mathrm{~km}$ distances (Figure 2). Depths determined from the two techniques also showed an increase at these places. These features along the profile correspond to seamount ${ }^{28}$ and Comorin ridge $^{29}$ in the south and north respectively.

\section{M2M2 profile}

This profile is about $1025 \mathrm{~km}$ long between $10^{\circ}$ to $0^{\circ} \mathrm{S}$ lat. and along $81^{\circ} \mathrm{E}$ long. The profile is divided into seven segments (Table 1).
The spectral and Werner depths to the top and bottom of the oceanic crust were determined from the unfiltered and filtered magnetic anomalies respectively. Table 1 shows all the depth values.

The magnetic basement depths obtained using spectral and Werner techniques were in the range $5.35-6.3 \mathrm{~km}$ and $5.2-6.5 \mathrm{~km}$ respectively. The deeper marker (Curie isotherm) as obtained from spectral studies was in the range $10.25-12.89 \mathrm{~km}$, whereas the Werner depths to the deeper boundary were in the range $9.0-13.0 \mathrm{~km}$.

A tentative crustal model was developed from these depths of spectral and Werner techniques (Figure 3). There was a good agreement between the depths obtained 


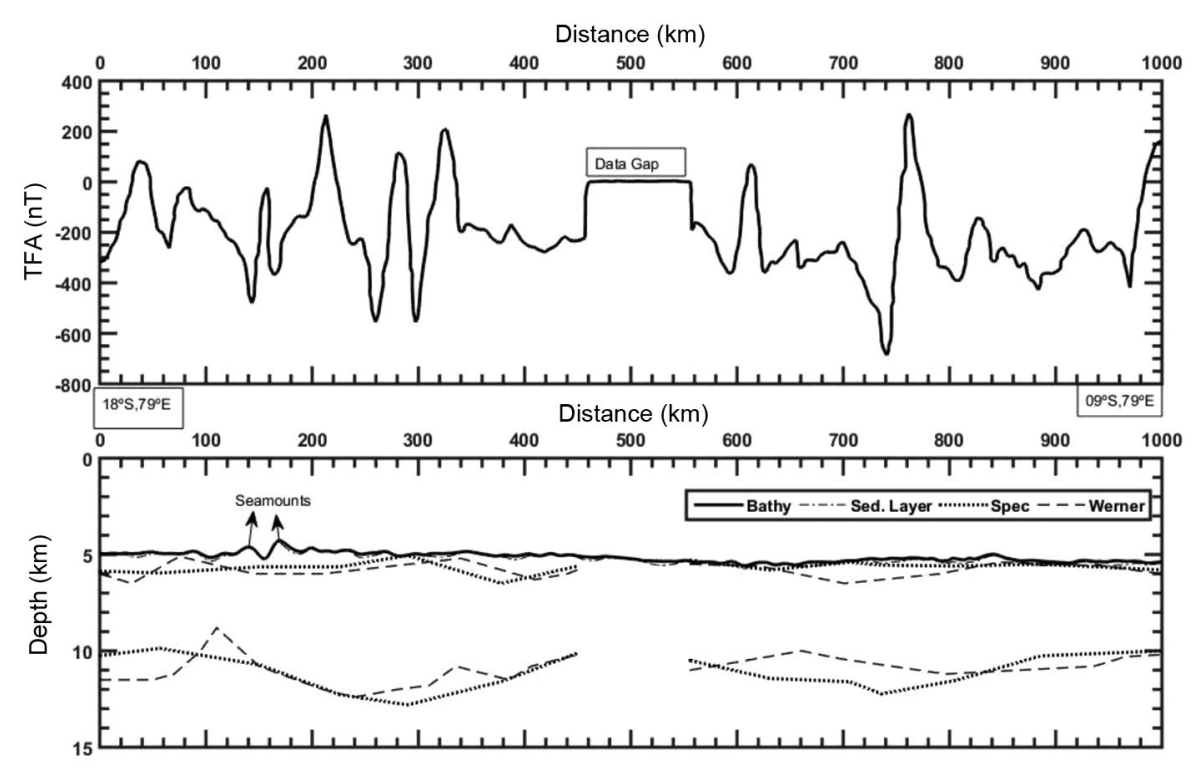

Figure 4. (Top) TFA along profile M3M3. (Bottom) Tentative crustal model derived from spectral and Werner deconvolution analysis along with bathymetry and sedimentary layers.

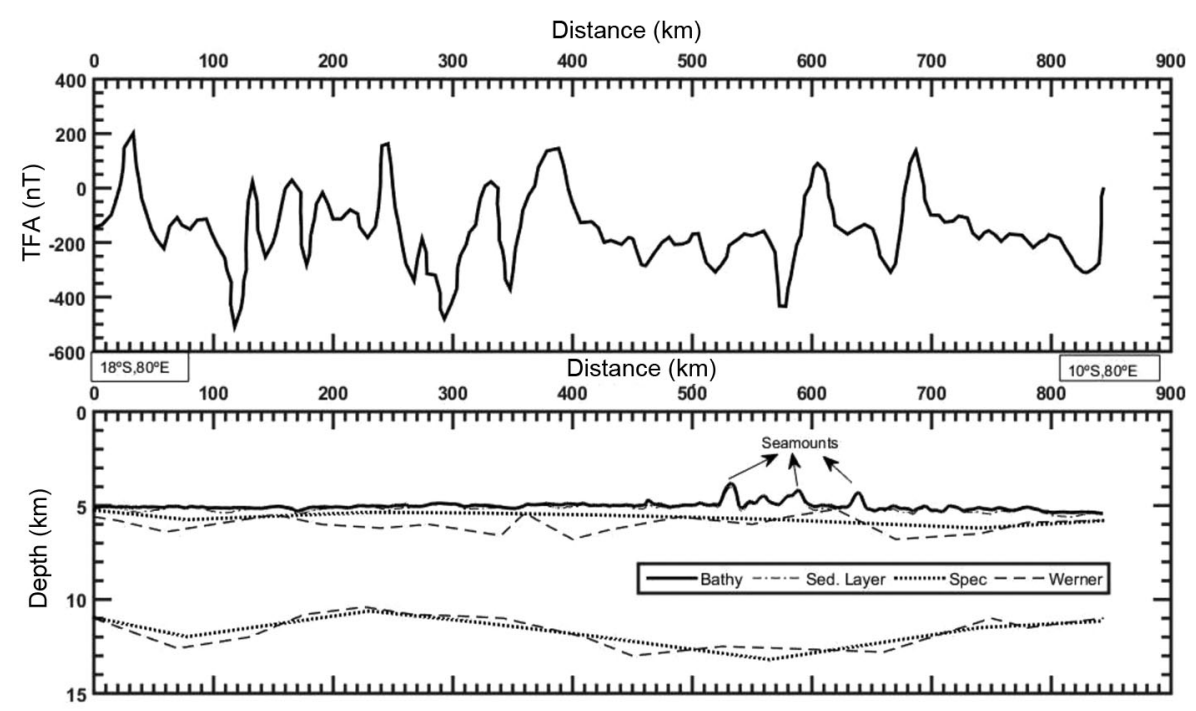

Figure 5. (Top) TFA along profile M4M4. (Bottom) Tentative crustal model derived from spectral and Werner deconvolution analysis along with bathymetry and sedimentary layers.

from spectral and Werner deconvolution techniques (Figure 3). The crests and troughs as inferred from this study (Table 1) were also in good agreement with the findings of Bull and Scrutton ${ }^{2}$ at a few locations.

\section{M3M3 profile}

This profile is about $999 \mathrm{~km}$ long and between $18^{\circ} \mathrm{S}$ to $9^{\circ} \mathrm{S}$ lat. and along $79^{\circ} \mathrm{E}$ long. The profile runs adjacent to $79^{\circ} \mathrm{E} \mathrm{FZ}$. It is divided into two sub-profiles, viz. M3M3$\mathrm{A}$ and M3M3-B. M3M3-A is $\sim 450 \mathrm{~km}$ long $\left(18^{\circ}-13.91^{\circ} \mathrm{S}\right.$ along $79^{\circ} \mathrm{E}$ ), while $\mathrm{M} 3 \mathrm{M} 3-\mathrm{B}$ is $\sim 440 \mathrm{~km}$ long $\left(12.91^{\circ}\right.$ $9^{\circ} \mathrm{S}$ along $79^{\circ} \mathrm{E}$ longitude).
The entire profile was divided into eight segments (Table 1). Table 1 shows the shallow and Curie isotherm depths obtained from the spectral and Werner deconvolution techniques. The depths to the magnetic basement determined from spectral and Werner techniques were in the range $5.2-6.5 \mathrm{~km}$ and $5.2-7.2 \mathrm{~km}$ respectively. Deeper marker (Curie isotherm) was in the range 9.87-12.8 km and $8.8-14 \mathrm{~km}$ as obtained using the spectral and Werner technique respectively. Table 1 gives the positions of crests and troughs observed along the profile M3M3. Crests were located at $17.3^{\circ} \mathrm{S}, 15.26^{\circ} \mathrm{S}$ and $9.5^{\circ} \mathrm{S}$; trough positions were observed at $16.7^{\circ} \mathrm{S}, 14.08^{\circ} \mathrm{S}$ (along the $79^{\circ} \mathrm{E}$ Indrani FZ). A tentative crustal model was prepared 

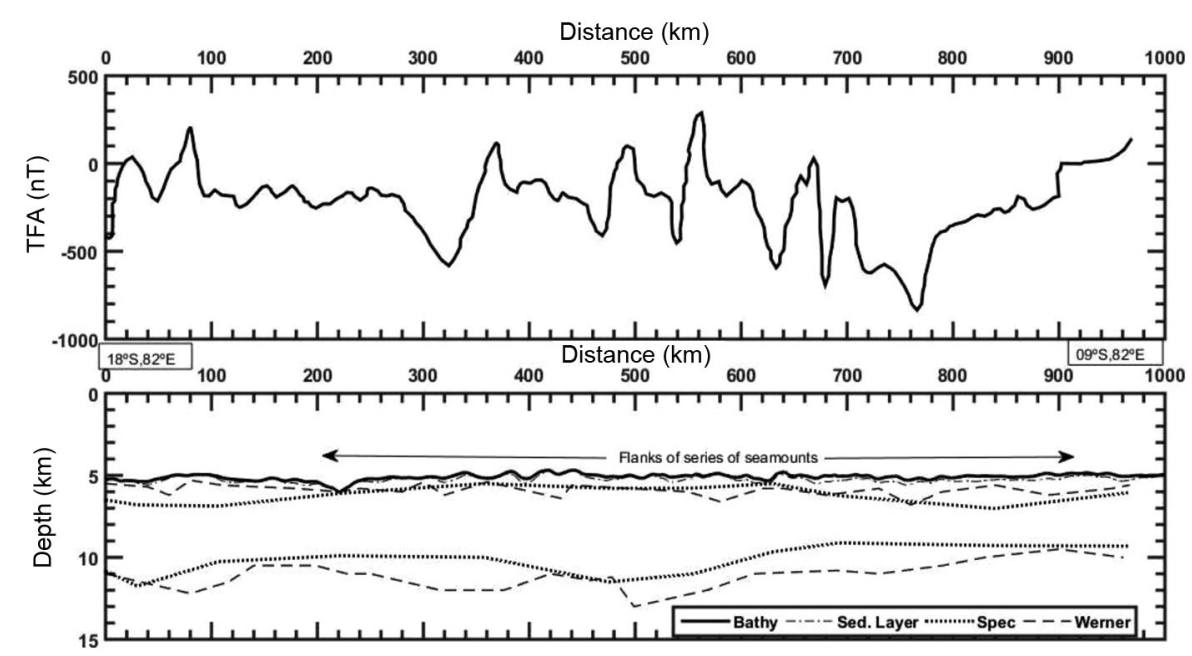

Figure 6. (Top) Total TFA along profile M6M6. (Bottom) Tentative crustal model derived from spectral and Werner deconvolution analysis along with bathymetry and sedimentary layers.

from these depths (Figure 4). The bathymetry, sediment thickness and magnetic basement layer showed sharp undulations at distances of 100-200 km (Figure 4). This may be due to a group of small seamounts present to the west of the M3M3 profile ${ }^{28}$.

\section{M4M4 profile}

This profile is between $18^{\circ} \mathrm{S}$ and $10^{\circ} \mathrm{S}$ lat. and along $80^{\circ} \mathrm{E}$

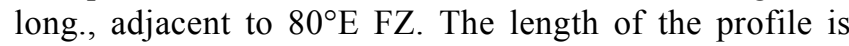
$843 \mathrm{~km}$. The entire profile was divided into six segments (Table 1). Depths to the top marker and bottom marker are given in Table 1 and Figure 5 shows the tentative magnetic crustal model.

The spectral depths to the top and bottom of the crust were in the range $5.36-6.2 \mathrm{~km}$ and $10.6-13.2 \mathrm{~km}$ respectively, whereas Werner depths to the top and bottom were in the range $5-6.8 \mathrm{~km}$ and $9.6-13.5 \mathrm{~km}$ respectively. The bathymetry also showed three humps between 453 and $637 \mathrm{~km}$, which may due to seamounts ${ }^{28}$. The magnetic basement depths also showed an increase at these locations.

\section{M6M6 profile}

The N-S transect M6M6 is $936 \mathrm{~km}$ long, it lies between $18^{\circ} \mathrm{S}$ and $9^{\circ} \mathrm{S}$ lat. and along $82^{\circ} \mathrm{E}$ long. The entire profile was divided into nine segments (Table 1).

The depths to the magnetic basement and Curie isotherm obtained from spectral and Werner techniques were in the range $5.3-6.3 \mathrm{~km}$ and $5.2-6.4 \mathrm{~km}$ while and the bottom depths (Curie isotherm) were in the range 9.9$12.96 \mathrm{~km}$ and $9.5-13 \mathrm{~km}$ respectively. The depth ranges obtained using both the techniques were almost the same (Figure 6 and Table 1).
The crest and trough points of profile M6M6 were identified from the data. Crests were located at $15.2^{\circ} \mathrm{S}$ and $12.2^{\circ} \mathrm{S}$ at depths of 5.5 and $5.1 \mathrm{~km}$ respectively. The trough point was located at $13.8^{\circ} \mathrm{S}$ and its depth was $6.4 \mathrm{~km}$ (Figure 6 and Table 1). The profile crosses a series of seamounts ${ }^{28}$.

\section{M7M7 profile}

The NS-trending profile M7M7 is $834 \mathrm{~km}$ long. It lies between $18^{\circ} \mathrm{S}$ and $10^{\circ} \mathrm{S}$ lat. and $83^{\circ} \mathrm{E}$ long. The entire profile was divided into seven segments (Table 1). Depths to the top and bottom as obtained from spectral and Werner analysis are shown in Table 1 and Figure 7 shows the tentative magnetic crustal model.

Along this profile bathymetry varied in the range 3.19$5.5 \mathrm{~km}$ with shallow depth of $3.19 \mathrm{~km}$ at a distance of $480 \mathrm{~km}$. The spectral depths to the magnetic basement were in the range $5.01-6.33 \mathrm{~km}$, whereas deeper magnetic horizon (Curie isotherm) was in the depth range 9.5$12.0 \mathrm{~km}$. The depths obtained from Werner technique also showed similar variation. There was a good agreement between depths obtained by spectral and Werner techniques (Figure 7).

This profile $\mathrm{M} 7 \mathrm{M} 7$ had a crest point at $13.7^{\circ} \mathrm{S}, 83^{\circ} \mathrm{E}$ and trough at $15.2^{\circ} \mathrm{S}, 83^{\circ} \mathrm{E}$. The topography, sediment and magnetic basement undulations may correspond to seamounts ${ }^{28}$. The Indira FZ runs between $3^{\circ} \mathrm{N}$ and $22^{\circ} \mathrm{S}$ lat. and along $83^{\circ} \mathrm{E}$ long.

\section{M8M8 profile}

The profile M8M8 between $18^{\circ} \mathrm{S}$ and $8^{\circ} \mathrm{S}$ lat. and along $84^{\circ} \mathrm{E}$ long. is $1105 \mathrm{~km}$ long. Along this profile also bathymetry was not uniform. Bathymetry showed crests 


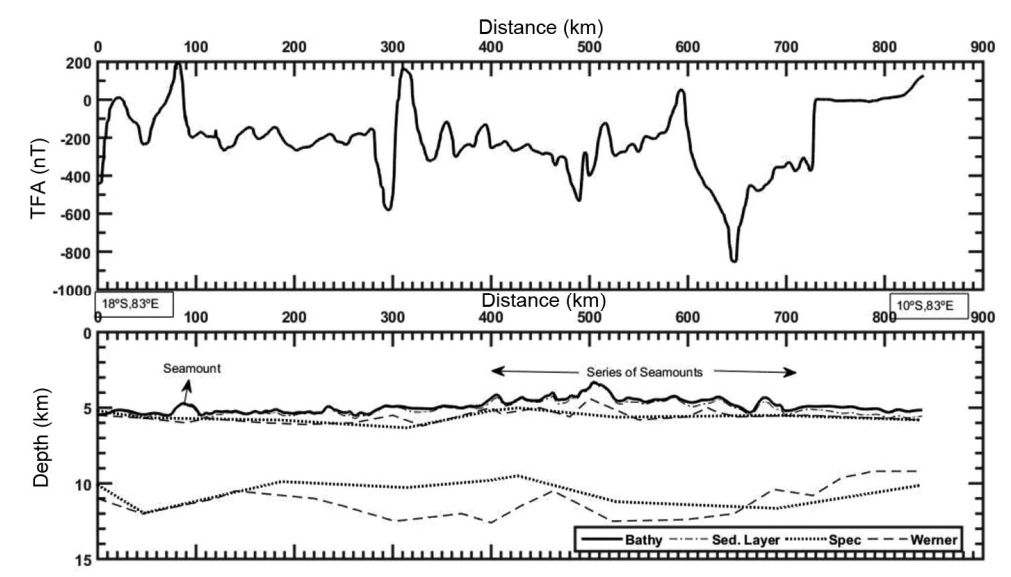

Figure 7. (Top) TFA along profile M7M7. (Bottom) Tentative crustal model derived from spectral and Werner deconvolution analysis along with bathymetry and sedimentary layers.

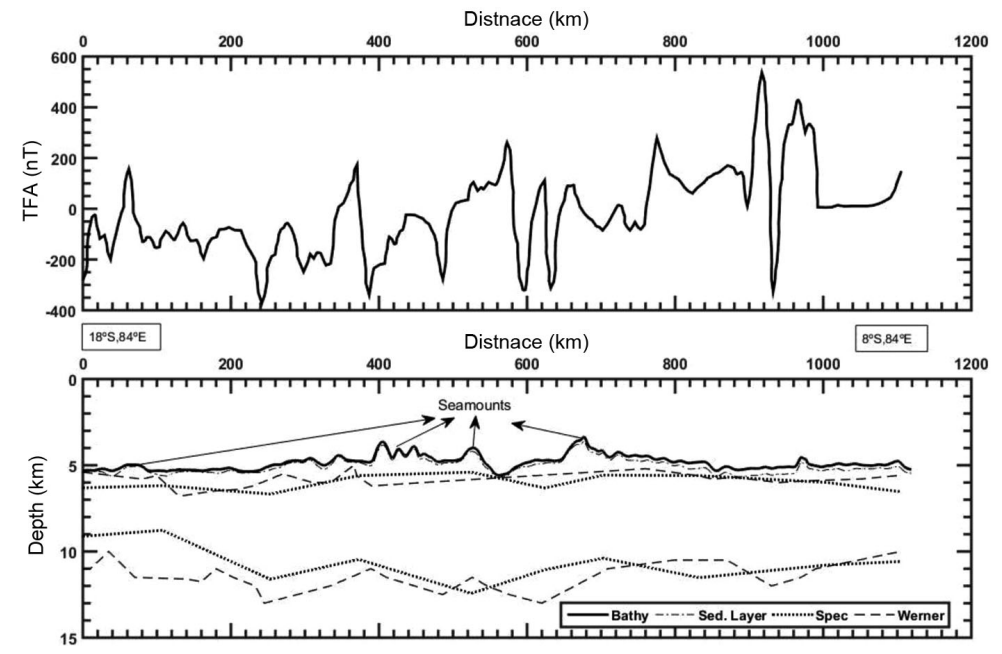

Figure 8. (Top) TFA along profile M8M8. (Bottom) Tentative crustal model derived from spectral and Werner deconvolution analysis along with bathymetry and sedimentary layers.

at $14.3^{\circ}$ and $13.8^{\circ} \mathrm{S}$. The entire profile was divided into eight segments (Table 1). Depths to the magnetic basement and Curie isotherm as obtained from spectral and Werner analysis are shown in Table 1 and Figure 8 shows the tentative magnetic crustal model.

The spectral depths to the top and bottom of the oceanic crust were in the range $5.0-6.67 \mathrm{~km}$ and $10.59-$ $12.84 \mathrm{~km}$ respectively, whereas Werner depths to the top showed a variation from 4.4 to $6.8 \mathrm{~km}$ and Curie isotherms were in the depth range $10.0-13.0 \mathrm{~km}$. There was a good agreement between the spectral and Werner depths (Figure 8). This profile crosses a series of seamounts $^{28}$.

\section{M9M9 profile}

This is the longest among all the nine profiles. It is oriented in SSW-NNE direction $\left(15^{\circ} 30^{\prime} \mathrm{S}, 77^{\circ} \mathrm{E}-6^{\circ} \mathrm{N}\right.$, $79^{\circ} \mathrm{E}$ ). The length of the profile is $2363 \mathrm{~km}$. The entire profile was divided into 14 segments. In the northern part
(1800-2000 km) the profile passes through the Comorin Ridge.

From spectral analysis, the depths to the top marker (magnetic basement) were found to be in the range 4.28$6.62 \mathrm{~km}$ and depth variation to the bottom interface (Curie isotherm) was found to be at 10.31-11.8 km. From the Werner deconvolution analysis, depth variations for shallow and deeper interfaces of the magnetic crust were in the range $4.4-6.8 \mathrm{~km}$ and $9.2-13.6 \mathrm{~km}$ respectively. The tentative crustal model is shown in Figure 9 and Table 1 gives the depth values.

\section{M10M10 profile}

Profile M10M10 runs along SSE to NNW $\left(7.5^{\circ} \mathrm{S}, 79^{\circ} \mathrm{E}\right.$ and $\left.3.5^{\circ} \mathrm{N}, 76^{\circ} \mathrm{E}\right)$. It has a length of $1288 \mathrm{~km}$. The entire profile was divided into seven segments (Table 1). The depths obtained from spectral and Werner techniques to the magnetic basement were in the range $3.5-6.056 \mathrm{~km}$ and $4.1-6.8 \mathrm{~km}$ respectively. The deeper marker 


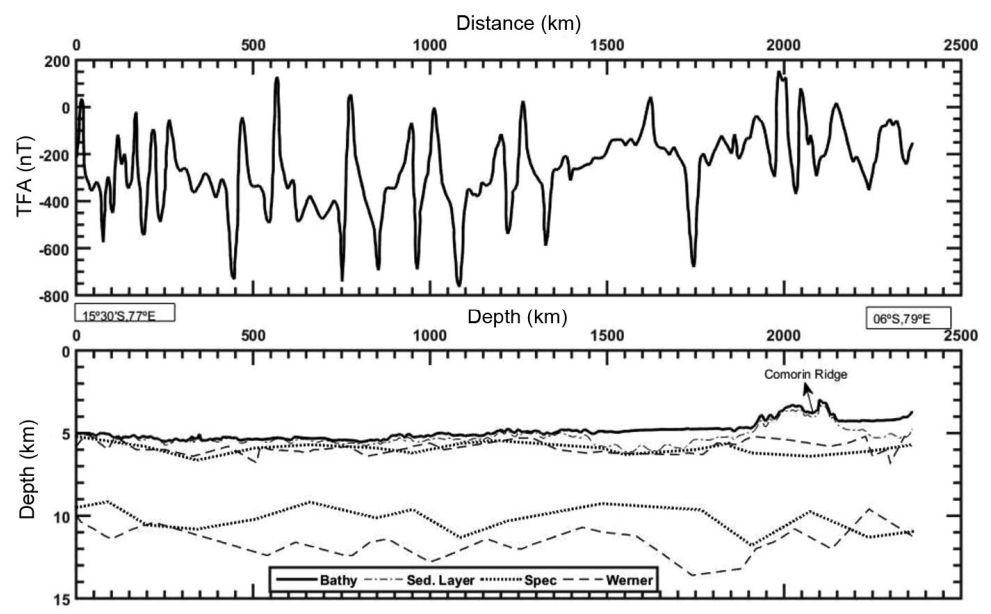

Figure 9. (Top) TFA along profile M9M9. (Bottom) Tentative crustal model derived from spectral and Werner deconvolution analysis along with bathymetry and sedimentary layers.

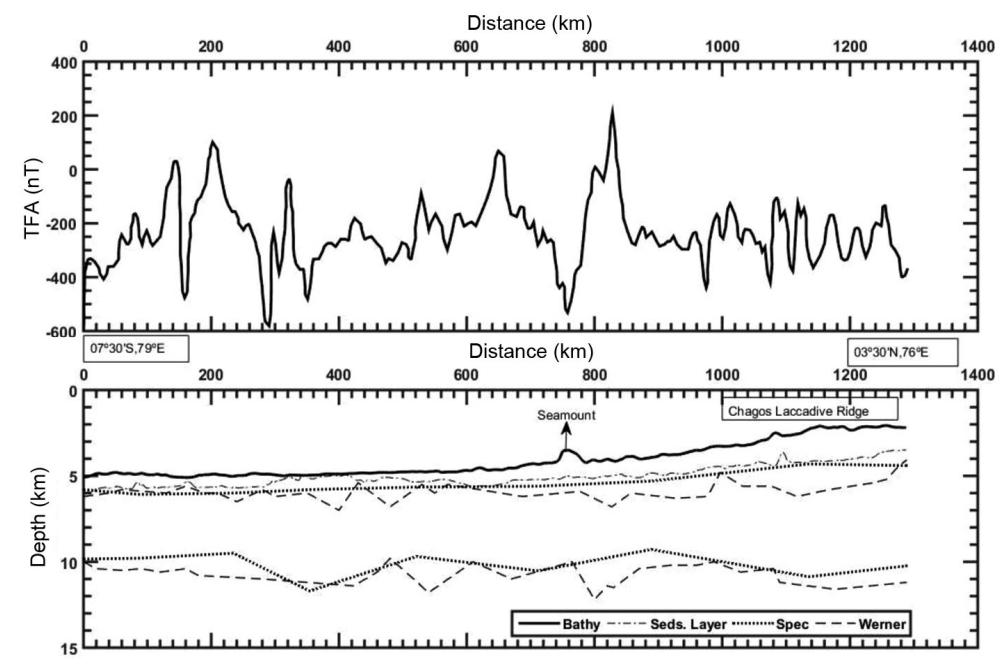

Figure 10. (Top) TFA along profile M10M10. (Bottom) Tentative crustal model derived from spectral and Werner deconvolution analysis along with bathymetry and sedimentary layers.

(Curie isotherm) as obtained from spectral studies varied in the range $9.3-12.54 \mathrm{~km}$, whereas the Werner depths to the deeper boundary varied in the range $8.8-$ $12.5 \mathrm{~km}$. The spectral and Werner depth sections were in good agreement. All the depth values are shown in Table 1 and Figure 10 shows the tentative crustal model.

The crest positions at $0.5^{\circ} \mathrm{S}, 76.8^{\circ} \mathrm{E}$ and trough position at $3.6^{\circ} \mathrm{E}, 77.6^{\circ} \mathrm{E}$ were inferred from the crustal model (Figure 10). Close to the northern end, the profile crosses a seamount and Chagos Laccadive ridge (CLR).

\section{Summary and conclusion}

The deformation zone of the CIO west of Ninety East Ridge dividing the Indian and Australian plates has been mapped up to $8^{\circ} \mathrm{S}$ from seismic studies. The crests and troughs of this deformation zone have been identified by numerous researchers. Here, we aimed to verify the possibility of extension of this deformation zone towards the south by interpreting magnetic anomalies along nine pro- files extending up to $18^{\circ} \mathrm{S}$. These magnetic anomalies have been analysed for age of the oceanic crust by numerous workers earlier. The pattern of the magnetic anomalies clearly indicates as due to different blocks of basaltic layer formed during different periods. The disturbance of this systematic pattern of anomalies at some locations indicates the deformation of these crustal blocks.

A preliminary interpretation of these magnetic profiles showed that spectral and Werner deconvolution methods yield varying depths to both shallow (magnetic basement) and deep (Curie isotherm/bottom of oceanic crust) markers. Figures 2-10 are plots of the tentative models. From these models, it was inferred that the crust must have been subjected to deformation even to the south of the deformation zone, identified up to $8^{\circ} \mathrm{S}$. There is close agreement between the depths obtained from spectral and Werner deconvolution techniques. From the crustal models, it was inferred that the variations the crustal thickness are indicative of intense deformation. From an integrated interpretation of longitudinal FZs, sea-floor topography, 
gravity and seismic data of the study area, several deformed crustal features have been identified ${ }^{30}$.

The results from the present study of magnetic data analysis are consistent with those of earlier studies based on seismic and earthquake data analysis ${ }^{2,3,6,12,15}$. The significance of this study is that there is good correlation between the depths obtained from two independent techniques. Spectral and Werner deconvolution techniques further authenticate the depths determined. Further, the study showed extension of the CIO deformation zone as evidenced by variation in the thickness of the crust (Figures 2-10) further south of $8^{\circ} \mathrm{S}$ lat. Detailed modelling of these anomalies using optimization iterative techniques would exactly reveal fine-scale deformation structures, and such a study is in progress.

1. Sykes, L. R., Seismicity of the Indian Ocean and a possible nascent island arc between Ceylon and Australia. J. Geophys. Res., 1970, 75, 5041-5055.

2. Bull, J. M. and Scrutton, R. A., Seismic reflection images of intraplate deformation, Central Indian Ocean, and their tectonic significance. J. Geol. Soc. London, 1992, 149, 955-966; doi:10.1144/ gsjgs.149.6.0955.

3. Bergman, E. A. and Solomon, S. C., Earthquake source mechanisms from body-waveform inversion and intraplate tectonics in the northern Indian Ocean. Phys. Earth Planet. Inter., 1985, 40, 1-23.

4. Cloetingh, S. and Wortel, R., Stress in the Indo-Australian plate Tectonophysics, 1986, 132, 49-67.

5. Curray, J. R., Emmel, F. J., Moore, D. G. and Raitt, R. W., Structure, tectonics and geological history of the Northeastern Indian Ocean. In The Ocean Basins and Margins (eds Nairn, A. E. and Stehli, F. G.), Plenum, New York, USA, 1982, vol. 6, pp. 399-450.

6. Krishna, K. S., Gopala Rao, D. and Neprochnov, Y. P., Formation of diapiric structure in the deformation zone, Central Indian Ocean: amodel from gravity and seismic reflection data. Proc. Indian Acad. Sci. (Earth Planet. Sci.), 2002, 111(1), 17-28.

7. Petroy, D. E. and Wiens, D. A., Historical seismicity and implications for diffuse plate convergence in the Northeast Indian Ocean. J. Geophys. Res., 1989, 94(B9), 12301-12319.

8. Stein, C. A. and Weissel, J. K., Constraints on the Central Indian Basin thermal structure from heat flow, seismicity and bathymetry. Tectonophysics, 1990, 176, 315-332.

9. Stein, C. A., Cloetingh, S. and Wortel, R., Seasat derived gravity data constraints on stress and deformation in the northeastern Indian Ocean. Geophys. Res. Lett., 1989, 16, 823-826.

10. Neprochnov, Y. P., Levchenko, O. V., Merklin, L. R. and Sedov, V. V., The structure and tectonics of the intraplate deformation area in the Indian Ocean. Tectonophysics, 1988, 156, 89-106; https://doi.org/10.1016/0040-1951(88)90285-5.

11. Bull, J. M., Structural style of intra-plate deformation, Central Indian Ocean Basin: evidence for the role of fracture zones. Tectonophysics, 1990, 184, 213-228.

12. Bull, J. M. and Scrutton, R. A., Fault reactivation in the central Indian Ocean and the rheology of oceanic lithosphere. Nature, 1990, 344, 855-858; doi:10.1038/344855a0.

13. Weissel, J. K., Anderson, R. N. and Geller, C. A., Deformation of the Indo-Australian Plate. Nature, 1980, 287, 284-291.

14. Geller, C. A., Weissel, J. K. and Anderson, R. N., Heat transfer and intraplate deformation in the Central Indian Ocean. J. Geophys. Res., 1983, 88, 1018-1032.
15. Krishna, K. S. et al., Tectonic model for the evolution of oceanic crust in the northeastern Indian Ocean from the Late Cretaceous to the Early Tertiary. J. Geophys. Res., 1995, 100, 20011-20024.

16. DeMets, C., Gordon, R. G. and Argus, D. F., Intraplate deformation and closure of the Australia-Antarctica-African Plate circuit. J. Geophys. Res., 1988, 93, 11877-11897.

17. Gordon, R. G., DeMets, C. and Argus, D. F., Kinematic constraints on distributed lithospheric deformation in the equatorial Indian Ocean from present motion between the Australian and Indian plates. Tectonics, 1990, 9(3), 409-422.

18. Royer, J. Y. and Chang, T., Evidence for relative motions between the Indian and Australian Plates during the last 20 m.y. from plate tectonic reconstructions: implications for the deformation of the Indo-Australian Plate. J. Geophys. Res., 1991, 96(B11), 1177911802.

19. Bull, J. M., Martinod, J. and Davy, P., Buckling of the oceanic lithosphere from geophysical data and experiments. Tectonics, 1992, 11(3), 537-548.

20. Krishna, K. S., Ramana, M. V., Gopala Rao, D., Murthy, K. S. R., Malleswara Rao, M. M., Subrahmanyam, V. and Sarma, K. V. L. N. S., Periodic deformation of oceanic crust in the Central Indian Ocean. J. Geophys. Res., 1998, 103, 17859-17875; doi:10.1029/ 98JB00078.

21. Anderson, R. N. and Langseth, M. G., The mechanisms of heat transfer through the floor of the Indian Ocean. J. Geophys. Res., 1977, 82(23), 3391-3409.

22. Mukhopadhyay, R. and Nath, B. N., Influence of seamount topography on the local facies variation in ferromanganese deposits in the Indian Ocean. Deep-Sea Res., 1988, 35, 1431-1436.

23. Siddiquie, H. N., Das Gupta, D. R., Sen Gupta, N. R., Shrivastava, P. C. and Mallik, T. K., Manganese iron nodules from the Indian Ocean. Indian J. Mar. Sci., 1978, 7, 239-253.

24. Cronan, D. S., Underwater Minerals, Academic Press, Oxford, UK, 1980, p. 363.

25. Mukhopadhyay, R., Iyer, S. D. and Ghosh, A. K., The Indian Ocean nodule field: petrotectonic evolution and ferromanganese deposits. Earth Sci. Rev., 2002, 60, 67-130.

26. Subrahmanyam, M. and Gebissa, F. T., Performance evaluation of spectral analysis and Werner deconvolution interpretation techniques in magnetic method. J. Appl. Geophys., 2017, 138, 102-113.

27. Rajendran, S., Magnetic and gravity studies over some parts of Central Indian Ocean with special reference to the deformed zone. $\mathrm{PhD}$ thesis submitted to the Andhra University, Visakhapatnam, 1995.

28. Mukhopadhyay, R. and Khadge, N. H., Seamounts in the Central Indian Ocean Basin: indicators of the Indian plate movement. Proc. Indian Acad. Sci. (Earth Planet. Sci.), 1990, 99(3), 357365; https://doi.org/10.1007/BF02841864.

29. Sreejith, K. M., Krishna, K. S. and Bansal, A. R., Structure and isostatic compensation of the Comorin Ridge, north central Indian Ocean. Geophys. J. Int., 2008, 175(2), 729-741; https://doi.org/ 10.1111/j.1365-246X.2008.03905.x.

30. Gopala Rao, D., Krishna, K. S., Neprochnov, Y. P. and Grinko, B. N., Satellite gravity anomalies and crustal features of the Central Indian Ocean Basin. Curr. Sci., 2004, 86(7), 948-957.

ACKNOWLEDGEMENT. R.D.B. thanks the Indian Institute of Geomagnetism, Mumbai for financial support.

Received 14 October 2019; revised accepted 26 August 2020

doi: $10.18520 / \mathrm{cs} / \mathrm{v} 119 / \mathrm{i} 9 / 1526-1539$ 